\title{
Generation of symmetry coordinates for crystals using multiplier representations of the space groups
}

\author{
Hansen, Flemming Yssing
}

Published in:

Physical Review B

Link to article, DOI:

10.1103/PhysRevB.18.4015

Publication date:

1978

Document Version

Publisher's PDF, also known as Version of record

Link back to DTU Orbit

Citation (APA):

Hansen, F. Y. (1978). Generation of symmetry coordinates for crystals using multiplier representations of the space groups. Physical Review B, 18(8), 4015-4038. https://doi.org/10.1103/PhysRevB.18.4015

\section{General rights}

Copyright and moral rights for the publications made accessible in the public portal are retained by the authors and/or other copyright owners and it is a condition of accessing publications that users recognise and abide by the legal requirements associated with these rights.

- Users may download and print one copy of any publication from the public portal for the purpose of private study or research.

- You may not further distribute the material or use it for any profit-making activity or commercial gain

- You may freely distribute the URL identifying the publication in the public portal

If you believe that this document breaches copyright please contact us providing details, and we will remove access to the work immediately and investigate your claim 


\title{
Generation of symmetry coordinates for crystals using multiplier representations of the space groups
}

\author{
Flemming Yssing Hansen \\ Fysisk-Kemisk Institut, The Technical University of Denmark, DK 2800 Lyngby, Denmark
}

(Received 27 April 1977)

\begin{abstract}
Symmetry coordinates play an important role in the normal-mode calculations of crystals. It is therefore of great importance to have a general method, which may be applied for any crystal at any wave vector, to generate these. The multiplier representations of the space groups as given by Kovalev and the projectionoperator technique provide a basis for such a method. The method is illustrated for the nonsymmorphic $D_{3}^{6}$ space group, and the theoretical background for the representations of space groups in general is reviewed and illustrated on the example above. It is desirable to perform the projection of symmetry coordinates in such a way that they may be used for as many wave vectors as possible. We discuss how to achieve this goal. The detailed illustrations should make it simple to apply the theory in any other case.
\end{abstract}

\section{INTRODUCTION}

Group-theoretical arguments are of great importance within the field of lattice-dynamical calculations and have been applied for many years.

If the potential energy of a crystal is expressed in terms of symmetry coordinates, which are displacement patterns of the constituent atoms spanning the group of the wave vectors, then the dynamical matrix will be on block form, as symmetry coordinates of different symmetry do not couple. The group of the wave vector is the space group, whose purely rotational elements leave the wave vector invariant (modulo $2 \pi$ times a translation vector of the reciprocal space). This may be used to check if the dynamical matrix is of the correct symmetry, because a wrong blocking will indicate that there are some mistakes in the setup of the problem. To avoid these problems in normalmode calculations, we have written a computer code ${ }^{1}$ which automatically on the basis of grouptheoretical arguments generates a dynamical matrix of the correct symmetry from an arbitrary input of coordinates and force constants for any lattice-vibrational problem. The block diagonalization of the dynamical matrix is often of great importance in the analysis of the effects of the various force constants in the model, especially when so-called symmetry-adapted coordinates ${ }^{2}$ are used in the definition of the force field. Also, the evaluation of the symmetry of the eigenvectors is simple and of great importance, for instance, when various branches are crossing and selection rules may be established. ${ }^{3}$

Recent excellent review articles by Maradudin and Vosko ${ }^{4}$ and Warren ${ }^{5}$ discuss the subject and give a survey of the historic development within the field. They show that the use of the so-called multiplier or ray representations of the 32 crystallo- graphic point groups provides a general basis for the description of the symmetry coordinates. This can be used as easily when the wave vector is inside the Brillouin zone, or when it is on the surface of the Brillouin zone, for symmorphic as well as for nonsymmorphic crystals. The method is, therefore, superior to previous methods ${ }^{4}$ which were problematic, in particular, for wave vectors at the Brillouin-zone boundary for crystals belonging to the nonsymmorphic space groups, that is to space groups among whose elements screw axes and/or glide planes are found. Kovalev seems to have been the first one to apply the multiplier representations of space groups and has published a book ${ }^{7}$ in which are tabulated the irreducible multiplier representations of the 230 space groups for many directions in the Brillouin zones. These tables are very useful and have made the application of multiplier representations practical for the construction of symmetry coordinates.

Hurley ${ }^{6}$ has also published a complete set of representations of the space groups based on multiplier representations of the point groups, but these are not found to be as easy to use as the ones given by Kovalev, although as pointed out by Warren, ${ }^{5}$ some mistakes may be found in the tables of the latter.

Owing to the importance of knowing the symmetry coordinates for a given crystal at any wave vector $\overrightarrow{\mathrm{k}}$, it is of great value to have a general method which may be applied without modifications to generate these. The multiplier representations of the space groups as tabulated by Kovalev provide a basis for such a method which is simple to apply. It was felt that a general presentation of the background for the method was highly desirable and useful so the outline of the paper is as follows. In Sec. II we present the theoretical background for the representations of the space groups. Our 
treatment differs somewhat from those cited above $^{4,5}$ and it is based on the one given by Koster, ${ }^{8}$ extended to include a natural introduction of multiplier representations of the point groups. A discussion of the basis sets which span these representations is also given. The developments in this section suppose a basic knowledge of group theory, which may be obtained from many sources, for instance Refs. 9-11. In Sec. III we illustrate in detail the development given in Sec. II using as an example the nonsymmorphic space group $D_{3}^{6}$ of trigonal selenium. The section is divided into three parts. In Sec. III A we illustrate the representation of the space-group elements for various wave vectors. In Sec. III B we show how a multiplier representation is obtained from an ordinary point-group representation. This is of importance since Kovalev's tables are not complete and suffer from misprints and errors. In Sec. III C we illustrate the generation of symmetry coordinates for different wave vectors and discuss how the projection ought to be done in order to obtain symmetry coordinates which may be used for as many points in reciprocal space as possible. From this example it should be a simple matter to consider any other crystal at any wave vector.

\section{BASIC THEORY}

The atoms in an infinite crystal are arranged in a regular pattern. This means, that there is a finite region in space (the unit cell), which is repeated in all directions. One may say, that the atoms span a lattice in space. Call the basis vectors of such a lattice $\vec{a}_{1}, \vec{a}_{2}, \vec{a}_{3}$. Then a primitive translation

$$
\overrightarrow{\mathrm{T}}_{\alpha}=\alpha_{1} \overrightarrow{\mathrm{a}}_{1}+\alpha_{2} \overrightarrow{\mathrm{a}}_{2}+\alpha_{3} \overrightarrow{\mathrm{a}}_{3} \quad\left(\alpha_{1}, \alpha_{2}, \alpha_{3} \text { are integers }\right)
$$

takes the space into itself, and the group of all primitive translations constitutes according to the defiinition $^{9}$ a group $T . T$ is an example of a space group, whose representation is particularly simple, since all irreducible representations are one dimensional, as we will see in a moment. In the Koster ${ }^{8}$ notation, an element of this group is $\left[\epsilon \mid \overrightarrow{\mathrm{T}}_{\alpha}\right]$, where $\epsilon$ (the rotational part of the operation) is the identity and $\overrightarrow{\mathrm{T}}_{\alpha}$ is given by Eq. (2.1). T is an infinite group, because there is an infinite number of translations (2.1). Also, $T$ is an Abelian group, ${ }^{9}$ as

$$
\begin{aligned}
{\left[\epsilon \mid \overrightarrow{\mathrm{T}}_{\alpha}\right]\left[\epsilon \mid \overrightarrow{\mathrm{T}}_{\alpha^{\prime}}\right] } & =\left[\epsilon \mid \epsilon \overrightarrow{\mathrm{T}}_{\alpha^{\prime}}+\overrightarrow{\mathrm{T}}_{\alpha}\right]=\left[\epsilon \mid \overrightarrow{\mathrm{T}}_{\alpha^{\prime}}+\overrightarrow{\mathrm{T}}_{\alpha}\right] \\
& =\left[\epsilon \mid \overrightarrow{\mathrm{T}}_{\alpha^{\prime}}\right]\left[\epsilon \mid \overrightarrow{\mathrm{T}}_{\alpha}\right],
\end{aligned}
$$

which again implies that all irreducible representations are one dimensional, because only in that case the order of the symmetry operations like in (2.2) is immaterial. We are going to deal with finite groups, so in order to make the translation group $T$ finite, we use the following expedient. It consists of imposing periodic boundary conditions, i.e., we put

$$
\left[\epsilon \mid \overrightarrow{\mathrm{a}}_{1}\right]^{N}=\left[\epsilon \mid \overrightarrow{\mathrm{a}}_{2}\right]^{N}=\left[\epsilon \mid \overrightarrow{\mathrm{a}}_{3}\right]^{N}=[\epsilon \mid \overrightarrow{0}]
$$

for some large but finite $N$. This means that we consider a space filled with exactly the same crystals, each with $N^{3}$ unit cells. In practice we always consider finite crystals, so there will never be an infinite number of translations leaving the crystal invariant. However, when $N$ is large enough the boundary effects will be unimportant. The representations of the finite translation group are now easy to obtain. Firstly, we remember, that the representation matrices are one dimensional. This means that $[\epsilon \mid \overrightarrow{0}]=1$. Secondly, Eq. (2.3) gives

$$
\left[\underline{D}\left(\left[\epsilon \mid \vec{a}_{1}\right]\right)\right]^{N}=1,
$$

where $D\left(\left[\epsilon \mid \vec{a}_{1}\right]\right)$ denotes the representation matrix of the operation $\left[\epsilon \mid \vec{a}_{1}\right]$, which here is a $(1 \times 1)$ matrix. There are $N$ roots of Eq. (2.4), and they are

$$
\begin{aligned}
& \underline{D}\left(\left[\epsilon \mid \overrightarrow{\mathrm{a}}_{1}\right]\right)=\exp \left(2 i \pi p_{1} / N\right), \\
& p_{1}=0,1,2, \ldots, N-1 .
\end{aligned}
$$

For translations along $\vec{a}_{2}$ and $\vec{a}_{3}$ one finds, as in (2.5a),

$$
\begin{aligned}
\underline{D}\left(\left[\epsilon \mid \overrightarrow{\mathrm{a}}_{2}\right]\right) & =\exp \left(2 i \pi p_{2} / N\right), \\
p_{2} & =0,1, \ldots, N-1, \\
\underline{D}\left(\left[\epsilon \mid \overrightarrow{\mathrm{a}}_{3}\right]\right) & =\exp \left(2 i \pi p_{3} / N\right), \\
p_{3} & =0,1, \ldots, N-1 .
\end{aligned}
$$

From (2.2) and (2.5a) we now deduce, that the representation of a given primitive translation operation $\overrightarrow{\mathrm{T}}_{\alpha}$ is given by

$$
\begin{aligned}
\underline{D}\left(\left[\epsilon \mid \overrightarrow{\mathrm{T}}_{\alpha}\right]\right) & =\underline{D}\left(\left[\epsilon \mid \alpha_{1} \overrightarrow{\mathrm{a}}_{1}\right]\right) \underline{D}\left(\left[\epsilon \mid \alpha_{2} \overrightarrow{\mathrm{a}}_{2}\right]\right) \underline{D}\left(\left[\epsilon \mid \alpha_{3} \overrightarrow{\mathrm{a}}_{3}\right]\right)=\underline{D}\left(\left[\epsilon \mid \overrightarrow{\mathrm{a}}_{1}\right]\right)^{\alpha_{1}} \underline{D}\left(\left[\epsilon \mid \overrightarrow{\mathrm{a}}_{2}\right]\right)^{\alpha_{2}} \underline{D}\left(\left[\epsilon \mid \overrightarrow{\mathrm{a}}_{3}\right]\right)^{\alpha_{3}} \\
& =\exp \left(\frac{2 i \pi p_{1} \alpha_{1}}{N}\right) \exp \left(\frac{2 i \pi p_{2} \alpha_{2}}{N}\right) \exp \left(\frac{2 i \pi p_{3} \alpha_{3}}{N}\right)=\exp \left[2 i \pi\left(\frac{p_{1}}{N} \alpha_{1}+\frac{p_{2}}{N} \alpha_{2}+\frac{p_{3}}{N} \alpha_{3}\right)\right] .
\end{aligned}
$$


When we define the reciprocal-lattice vectors $\vec{b}_{1}$, $\vec{b}_{2}, \vec{b}_{3}$ in the usual way

$$
\overrightarrow{\mathrm{b}}_{i} \cdot \overrightarrow{\mathrm{a}}_{j}=2 \pi \delta_{i j}, \quad i, j=1,2,3 .
$$

we see from (2.6) that

$$
\underline{D}\left(\left[\epsilon \mid \overrightarrow{\mathrm{T}}_{\alpha}\right]\right)=\exp \left(i \overrightarrow{\mathrm{k}} \cdot \overrightarrow{\mathrm{T}}_{\alpha}\right),
$$

where

$$
\overrightarrow{\mathrm{k}}=\frac{p_{1}}{N} \overrightarrow{\mathrm{b}}_{1}+\frac{p_{2}}{N} \overrightarrow{\mathrm{b}}_{2}+\frac{p_{3}}{N} \overrightarrow{\mathrm{b}}_{3}=k_{1} \overrightarrow{\mathrm{b}}_{1}+k_{2} \overrightarrow{\mathrm{b}}_{2}+k_{3} \overrightarrow{\mathrm{b}}_{3} .
$$

The representations are of course irreducible, because they are one dimensional. Thus, from (2.5a) and $(2.5 \mathrm{~b})$ and $(2.8)$ we see that there is one ir reducible representation for each value of $\vec{k}$, a total of $N^{3}$ in all. As $N$ becomes very large, the allowed values of $\overrightarrow{\mathrm{k}}$ fill out the parallelepiped spanned by the vectors $\overrightarrow{\mathrm{b}}_{1}, \overrightarrow{\mathrm{b}}_{2}, \overrightarrow{\mathrm{b}}_{3}$, except for the surfaces $k_{1}=1, k_{2}=1$, and $k_{3}=1$. Any reciprocal vector $\overrightarrow{\mathrm{k}}$ may be expressed as

$$
\overrightarrow{\mathrm{k}}=\overrightarrow{\mathrm{k}}_{0}+\overrightarrow{\mathrm{K}}
$$

where $\vec{k}_{0}$ is inside the fundamental parallelepiped or on the surface $k_{1}=0, k_{2}=0$, or $k_{3}=0 . \overrightarrow{\mathrm{K}}$ is zero or given by

$$
\overrightarrow{\mathrm{K}}=q_{1} \overrightarrow{\mathrm{b}}_{1}+q_{2} \overrightarrow{\mathrm{b}}_{2}+q_{3} \overrightarrow{\mathrm{b}}_{3},
$$

where $\left(q_{1}, q_{2}, q_{3}\right)$ are integers, and this is a lattice vector in reciprocal space. From (2.8), (2.10), and (2.11) it is clear, that a $\vec{k}$ vector outside the fundamental parallelepiped does not give rise to any new irreducible representations, because

$$
\begin{aligned}
\exp \left(i \overrightarrow{\mathrm{k}} \cdot \overrightarrow{\mathrm{T}}_{\alpha}\right) & =\exp \left[i\left(\overrightarrow{\mathrm{k}}_{0}+\overrightarrow{\mathrm{K}}\right) \cdot \overrightarrow{\mathrm{T}}_{\alpha}\right] \\
& =\exp \left(i \overrightarrow{\mathrm{k}}_{0} \cdot \overrightarrow{\mathrm{T}}_{\alpha}\right),
\end{aligned}
$$

which is one of the $N^{3}$ already known representations. The fundamental parallelepiped is no more than the well-known Brillouin zone in reciprocal space.

Consider now a space group $G$, which besides the translation operations includes elements with rotational operations and eventually also fractional translations. An element from such a group is written $\left[S_{i} \mid \vec{t}_{i}\right]$, where $S_{i}$ is the rotation operation and $\vec{t}_{i}$ is a translation vector (primitive or fractional) given in direct space.

It is easy to show, that the translation group $T$ is an invariant subgroup of the group $G$. For any element $\left[\epsilon \mid \vec{T}_{\alpha}\right]$ of the translation group and for any element $\left[S_{i} \mid \vec{t}_{i}\right]$ of the space group $G$, we find for the conjugate elements to the elements of the group $T$

$$
\begin{aligned}
{\left[S_{i} \mid \overrightarrow{\mathrm{t}}_{i}\right]^{-1}\left[\epsilon \mid \overrightarrow{\mathrm{T}}_{\alpha}\right]\left[S_{i} \mid \overrightarrow{\mathrm{t}}_{i}\right] } & =\left[S_{i}^{-1} \mid-S_{i}^{-1} \overrightarrow{\mathrm{t}}_{i}\right]\left[S_{i} \mid \overrightarrow{\mathrm{t}}_{i}+\overrightarrow{\mathrm{T}}_{\alpha}\right] \\
& =\left[\epsilon \mid S_{i}^{-1} \overrightarrow{\mathrm{t}}_{i}+S_{i}^{-1} \overrightarrow{\mathrm{T}}_{\alpha}-S_{i}^{-1} \overrightarrow{\mathrm{t}}_{i}\right] \\
& =\left[\epsilon \mid S_{i}^{-1} \overrightarrow{\mathrm{T}}_{\alpha}\right]=\left[\epsilon \mid \overrightarrow{\mathrm{T}}_{\alpha^{\prime}}\right]
\end{aligned}
$$

for all $\left[S_{i} \mid \vec{t}_{i}\right]$ because the rotation of a lattice vector $\overrightarrow{\mathrm{T}}_{\alpha}$ generates another lattice vector $\overrightarrow{\mathrm{T}}_{\alpha}$. We have thus shown that all operations, which are conjugated to any primitive translation operation, are themselves primitive translation operations. This is just the criteria for $T$ being an invariant subgroup of $G$. $G$ may therefore be decomposed into cosets $^{9,10}$ of $T$ :

$$
\begin{aligned}
G & =T+g_{2} T+g_{3} T+\cdots g_{i s} T \\
& =T+T g_{2}+\cdots T g_{i s},
\end{aligned}
$$

$g_{i}$ being a shorthand notation for the elements $\left[S_{i} \mid \vec{t}_{i}\right]$, where $S_{i}$ is the rotational part of the operation; $\vec{t}_{i}$ is a fractional translation or zero and is the number of operations whose rotational parts are different from the identity. The last relation in (2.13) holds, because $T$ is an invariant subgroup, so the left and right cosets are identical.

A matrix representation of symmetry operations depends, of course, on the basis which is chosen for the representation. This basis may be vectors, displacement patterns of atoms in a crystal, or functions. In the following let us consider an $n$-dimensional representation, and let us designate a basis for this representation $\left(\psi_{1}, \ldots, \psi_{n}\right)$. It is for this purpose immaterial whether the basis is vectors, displacements, or functions, and we will return to the problem of how to find a suitable set. In the following we will see that the re exists some relations between the members of the basis set, and we will stress them as they are deduced.

We have seen that all irreducible representations of the translation group $T$ are one dimensional, which means that unless $n$ is equal to one, the matrices $D\left(\left[\epsilon \mid \overrightarrow{\mathrm{T}}_{\alpha}\right]\right)$ are reducible. This means again that there exists a basis set, so $D\left(\left[\epsilon \mid \overrightarrow{\mathrm{T}}_{\alpha}\right]\right)$ is a diagonal matrix. We assume that this is the case for our basis.

Proposition 1. The representation matrices for the pure translation operations are diagonal matrices.

We may represent any "function" $\psi$ in the space spanned by our basis set by its components $s_{i}$ of each member of the basis set:

$$
\psi=\left(\psi_{1}, \ldots, \psi_{n}\right)\left[\begin{array}{c}
s_{1} \\
\cdot \\
\cdot \\
\cdot \\
s_{n}
\end{array}\right]=\sum_{i} s_{i} \psi_{i} .
$$

The representation matrix $\underline{D}([S \mid \vec{t}])$ of the operation $[S \mid \overrightarrow{\mathrm{t}}]$ is now defined according to 


$$
[S \mid \overrightarrow{\mathrm{t}}]=\left(\psi_{1}, \ldots, \psi_{n}\right) \underline{D([S \mid \overrightarrow{\mathrm{t}}])}\left[\begin{array}{c}
s_{1} \\
\cdot \\
\cdot \\
\cdot \\
s_{n}
\end{array}\right] .
$$

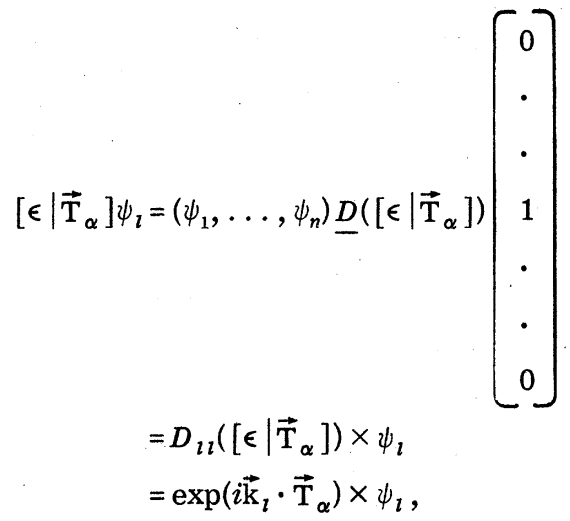

To illustrate the fact that the matrices $D([S \mid \vec{t}])$ depend on the basis set chosen, let us choose another basis set according to

$$
\left(\psi_{1}^{\prime}, \ldots, \psi_{n}^{\prime}\right)=\left(\psi_{1}, \ldots, \psi_{n}\right) \underline{Q}
$$

where the $\underline{Q}$ matrix relates the two basis sets. Then

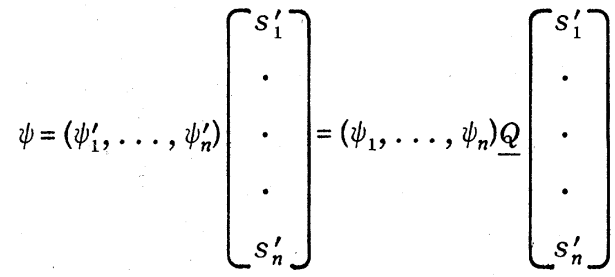

$$
=\left(\psi_{1}, \ldots, \psi_{n}\right)\left[\begin{array}{c}
s_{1} \\
\cdot \\
\cdot \\
s_{n}
\end{array}\right]
$$

and

$$
\begin{aligned}
& {[S \mid \overrightarrow{\mathrm{t}}] \psi=\left(\psi_{1}^{\prime}, \ldots, \psi_{n}^{\prime}\right) \underline{D}^{\prime}([s \mid \overrightarrow{\mathrm{t}}])\left[\begin{array}{c}
s_{1}^{\prime} \\
\cdot \\
\cdot \\
\cdot \\
s_{n}^{\prime}
\end{array}\right]} \\
& =\left(\psi_{1}, \ldots, \psi_{n}\right) \underline{\underline{Q}} \underline{D}^{\prime}([s \mid \overrightarrow{\mathrm{t}}]) \underline{Q}^{-1}\left[\begin{array}{c}
s_{1} \\
\cdot \\
\cdot \\
\cdot \\
s_{n}
\end{array}\right] \text {, }
\end{aligned}
$$

so

$$
\underline{D}([S \mid \overrightarrow{\mathrm{t}}])=\underline{Q} \underline{D}^{\prime}([S \mid \overrightarrow{\mathrm{t}}]) \underline{Q}^{-1} .
$$

This is the relation between the representation matrices in the two different basis sets $\left(\psi_{1}, \ldots, \psi_{n}\right)$ and $\left(\psi_{1}^{\prime}, \ldots, \psi_{n}^{\prime}\right)$. The effect of a primitive translation on a member of the basis, say $\psi_{l}$, is found to be because $D\left(\left[\epsilon \mid \overrightarrow{\mathrm{T}}_{\alpha}\right]\right)$ is diagonal. We also have assumed that the $(l, l)$ th element of $D\left(\left[\epsilon \mid \overrightarrow{\mathrm{T}}_{\alpha}\right]\right)$ corresponds to the irreducible representation of the translation group characterized by the reciprocal vector $\overrightarrow{\mathrm{k}}_{l}$. Let us then study the effect of $\left[\epsilon \mid \overrightarrow{\mathrm{T}}_{\alpha}\right]$ on the "function" $\psi_{l^{\prime}}=[S \mid \overrightarrow{\mathrm{t}}] \psi_{l}$. We find

$$
\begin{aligned}
{\left[\epsilon \mid \overrightarrow{\mathrm{T}}_{\alpha}\right] \psi_{l^{\prime}} } & =\left[\epsilon \mid \overrightarrow{\mathrm{T}}_{\alpha}\right][S \mid \overrightarrow{\mathrm{t}}] \psi_{l} \\
& =[S \mid \overrightarrow{\mathrm{t}}]\left[\epsilon \mid S^{-1} \overrightarrow{\mathrm{T}}_{\alpha}\right] \psi_{l},
\end{aligned}
$$

or written out,

$$
\begin{aligned}
& {\left[\epsilon \mid \overrightarrow{\mathrm{T}}_{\alpha}\right] \psi_{l^{\prime}}=\left(\psi_{1}, \ldots, \psi_{n}\right) \underline{D}\left(\left[\epsilon \mid \overrightarrow{\mathrm{T}}_{\alpha}\right]\right)} \\
& \times \underline{D}([S \mid \overrightarrow{\mathrm{t}}])\left[\begin{array}{c}
0 \\
\cdot \\
\cdot \\
\cdot \\
\cdot \\
0
\end{array}\right] \\
& =\left(\psi_{1}, \ldots, \psi_{n}\right) \underline{D}\left(\left[\epsilon \mid \overrightarrow{\mathrm{T}}_{\alpha}\right]\right) \\
& \times\left[\begin{array}{c}
D_{12}([S \mid \overrightarrow{\mathrm{t}}]) \\
\cdot \\
\cdot \\
D_{n l}([S \mid \overrightarrow{\mathrm{t}}])
\end{array}\right] \\
& =\left(\psi_{1}, \ldots, \psi_{n}\right)\left[\begin{array}{cc}
\exp \left(i \overrightarrow{\mathrm{k}}_{1} \cdot \overrightarrow{\mathrm{T}}_{\alpha}\right) D_{1 l}([S \mid \overrightarrow{\mathrm{t}}]) \\
\cdot \\
\cdot \\
\cdot \\
\exp \left(i \overrightarrow{\mathrm{k}}_{n} \cdot \overrightarrow{\mathrm{T}}_{\alpha}\right) D_{n l}([S \mid \overrightarrow{\mathrm{t}}])
\end{array}\right] \text {, }
\end{aligned}
$$

where we have called the diagonal elements of $\underline{D}\left(\left[\epsilon \mid \overrightarrow{\mathrm{T}}_{\alpha}\right]\right), e^{i \overrightarrow{\mathrm{k}}_{1} \cdot \overrightarrow{\mathrm{T}}_{\alpha}} \cdots e^{i \overrightarrow{\mathrm{k}}_{n} \cdot \overrightarrow{\mathrm{T}}_{\alpha}}$. Some of the $\overrightarrow{\mathrm{k}}_{i}$ vec- 
tors may be identical, as we will see in a moment. From the second relation in (2.14), we find

$$
\begin{aligned}
& {\left[\epsilon \mid \overrightarrow{\mathrm{T}}_{\alpha}\right] \psi_{t^{\prime}}=\left(\psi_{1}, \ldots, \psi_{n}\right) D([S \mid \overrightarrow{\mathrm{t}}])} \\
& \times \underline{D}\left(\left[\epsilon \mid S^{-1} \overrightarrow{\mathrm{T}}_{\alpha}\right]\right)\left[\begin{array}{c}
0 \\
\cdot \\
\cdot \\
1 \\
\cdot \\
0
\end{array}\right] \\
& =\left(\psi_{1}, \ldots, \psi_{n}\right)\left[\begin{array}{c}
D_{1 l}([s \mid \overrightarrow{\mathrm{t}}]) \\
\cdot \\
\cdot \\
\cdot \\
D_{n l}([S \mid \overrightarrow{\mathrm{t}}])
\end{array}\right] \\
& \times \exp \left[i \overrightarrow{\mathrm{k}}_{i} \cdot\left(S^{-1} \overrightarrow{\mathrm{T}}_{\alpha}\right)\right] \\
& =\left(\psi_{1}, \ldots, \psi_{n}\right)\left[\begin{array}{c}
\exp \left[i\left(S \overrightarrow{\mathrm{k}}_{\imath}\right) \cdot \overrightarrow{\mathrm{T}}_{\alpha}\right] D_{1 l}([S \mid \overrightarrow{\mathrm{t}}]) \\
\cdot \\
\cdot \\
\cdot \\
\cdot \\
\exp \left[i\left(S \overrightarrow{\mathrm{k}}_{\imath}\right) \cdot \overrightarrow{\mathrm{T}}_{\alpha}\right] D_{n l}([S \mid \overrightarrow{\mathrm{t}}])
\end{array}\right] \text {, }
\end{aligned}
$$

as

$$
\overrightarrow{\mathrm{k}}_{l} \cdot\left(S^{-1} \overrightarrow{\mathrm{T}}_{\alpha}\right)=\left(S \overrightarrow{\mathrm{k}}_{l}\right) \cdot \overrightarrow{\mathrm{T}}_{\alpha} \cdot
$$

From Eqs. (2.15) and (2.16) we may draw two important conclusions.

Proposition 2. Since at least one of the elements $D_{i l}$, say $D_{l, l}$, has to be different from zero (because we have assumed our irreducible representation to be $n$ dimensional), we see that if one of the diagonal elements in $\underline{D}\left(\left[\epsilon \mid \overrightarrow{\mathrm{T}}_{\alpha}\right]\right)$ is $\exp \left(i \overrightarrow{\mathrm{k}}_{l} \cdot \overrightarrow{\mathrm{T}}_{\alpha}\right)$, then the element $\exp \left[i\left(S \overrightarrow{\mathrm{k}}_{l}\right) \cdot \overrightarrow{\mathrm{T}}_{\alpha}\right]$ also has to appear once somewhere along the diagonal of $D\left(\left[\epsilon \mid \overrightarrow{\mathrm{T}}_{\alpha}\right]\right)$. Furthermore, if $\exp \left(i \overrightarrow{\mathrm{k}}_{l} \cdot \overrightarrow{\mathrm{T}}_{\alpha}\right)$ appears $\bar{d}$ times along the diagonal of $D\left(\left[\epsilon \mid \overrightarrow{\mathrm{T}}_{\alpha}\right]\right)$, then $\exp \left[i\left(S \overrightarrow{\mathrm{k}}_{l}\right) \cdot \overrightarrow{\mathrm{T}}_{\alpha}\right]$ also has to appea $\bar{r} d$ times. This is true for all elements of the group. (We do not consider the pure translational operations in this connection, of course.)

Proposition 3. The element $D_{j l}([S \mid \vec{t}])$ must be zero unless

$$
\exp \left(i \overrightarrow{\mathrm{k}}_{j} \cdot \overrightarrow{\mathrm{T}}_{\alpha}\right)=\exp \left[i\left(S \overrightarrow{\mathrm{k}}_{l}\right) \cdot \overrightarrow{\mathrm{T}}_{\alpha}\right]
$$

or

$$
\overrightarrow{\mathrm{k}}_{j}=S \overrightarrow{\mathrm{k}}_{l}+\overrightarrow{\mathrm{K}}_{q},
$$

where $\overrightarrow{\mathrm{K}}_{q}$ either is zero or a primitive vector in reciprocal space.

According to proposition 2 we may order the elements along the diagonal of $D\left(\left[\epsilon \mid \overrightarrow{\mathrm{T}}_{\alpha}\right]\right)$ in $d \times d$ blocks, where the diagonal elements in a block all are identical, and the different blocks are characterized by essential different $\vec{k}$ vectors. This ordering in blocks is, of course, equivalent to an ordering of the basis set in such a way, that the first $d$ members in the set transform like $\overrightarrow{\mathrm{k}}_{1}$ say, the next $d$ members transform like $\overrightarrow{\mathrm{k}}_{2}$, etc., under a primitive translation operation. Written out it looks like

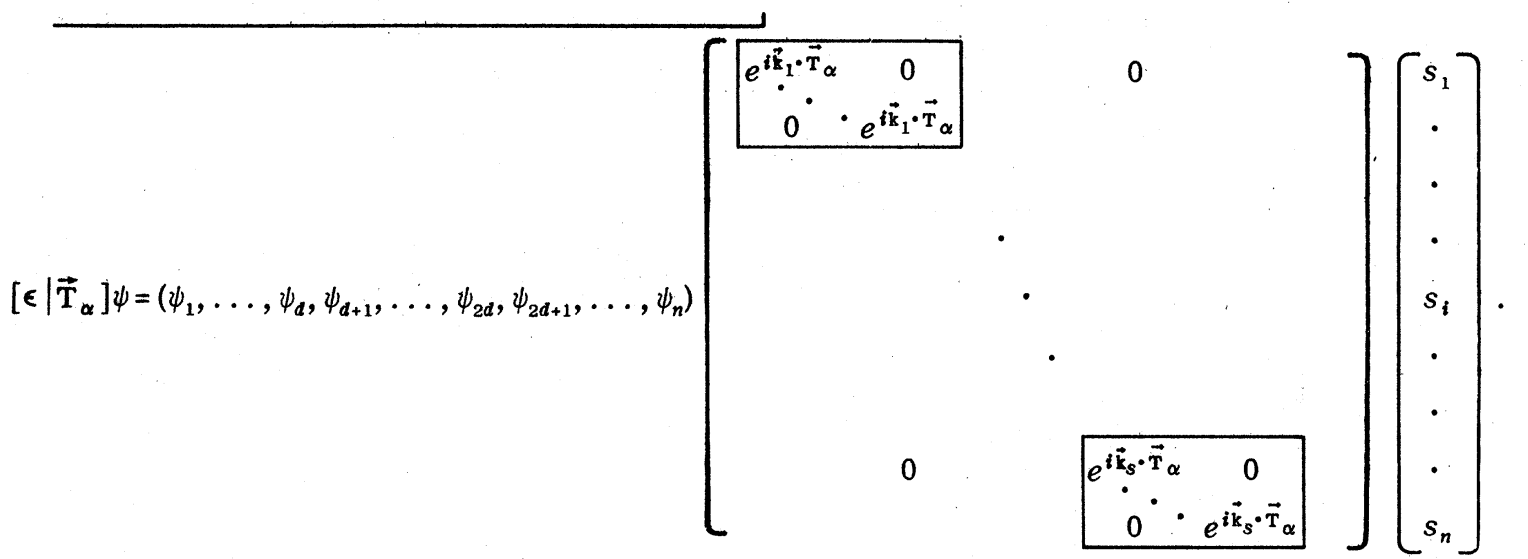

The number of blocks $s=n / d$ has to be an integer, of course, and we see that anyone of the first $d$ members of the basis set transform like $e^{i \overrightarrow{\mathrm{k}}_{1} \cdot \overrightarrow{\mathrm{T}}_{\alpha}}$, anyone member of the next $d$ members of the basis set transform like $e^{i \overrightarrow{\mathrm{k}}_{2} \cdot \vec{T}_{\alpha}}$, etc. $d$ greater than one means according to proposition 2 that there are elements in the group, whose rotational part $S$ has the property

$$
e^{i\left(S \overrightarrow{\mathrm{k}}_{1}\right) \cdot \overrightarrow{\mathrm{T}}_{\alpha}}=e^{i \overrightarrow{\mathrm{k}}_{1} \cdot \overrightarrow{\mathrm{T}}_{\alpha}}
$$

or 


$$
\begin{aligned}
& S \overrightarrow{\mathrm{k}}_{1}=\overrightarrow{\mathrm{k}}_{1}+\overrightarrow{\mathrm{K}}_{q}, \quad \overrightarrow{\mathrm{K}}_{q}=\left(q_{1} \overrightarrow{\mathrm{b}}_{1}+q_{2} \overrightarrow{\mathrm{b}}_{2}+q_{3} \overrightarrow{\mathrm{b}}_{3}\right) \\
& \left(q_{1}, q_{2}, q_{3} \text { are integers }\right) .
\end{aligned}
$$

The collection of such elements is said to form the group of $\vec{k}_{1}$. To distinguish these elements from others, let us designate members of the group of $\overrightarrow{\mathrm{k}}_{1}$ as

$$
\left[R_{i} \mid \overrightarrow{\mathrm{r}}_{i}\right]
$$

where

$$
R_{i} \overrightarrow{\mathrm{k}}_{1}=\overrightarrow{\mathrm{k}}_{1}+\overrightarrow{\mathrm{K}}_{q_{i}}
$$

It is easy to show that the elements $\left[R_{i} \mid \overrightarrow{\mathrm{r}}_{i}\right]$ in fact form a group, a subgroup of $G$. We find for any two elements of the group of $\overrightarrow{\mathrm{k}}_{1}, \mathrm{G}_{\overrightarrow{\mathrm{k}}_{1}}$ :

$$
\left[R_{j} \mid \overrightarrow{\mathrm{r}}_{j}\right]\left[R_{i} \mid \overrightarrow{\mathrm{r}}_{i}\right]=\left[R_{j} R_{i} \mid R_{j} \overrightarrow{\mathrm{r}}_{i}+\overrightarrow{\mathrm{r}}_{j}\right]=\left[R_{p} \mid \overrightarrow{\mathrm{r}}_{p}\right],
$$

where

$$
R_{j} \overrightarrow{\mathrm{k}}_{1}=\overrightarrow{\mathrm{k}}_{1}+\overrightarrow{\mathrm{K}}_{q_{j}}, \quad R_{i} \overrightarrow{\mathrm{k}}_{1}=\overrightarrow{\mathrm{k}}_{1}+\overrightarrow{\mathrm{K}}_{q_{i}}
$$

$\left[R_{p} \mid \overrightarrow{\mathrm{r}}_{p}\right]$ is an element of $G_{\overrightarrow{\mathrm{k}}_{1}}$ because

$$
\begin{aligned}
R_{p} \overrightarrow{\mathrm{k}}_{1}=R_{j} R_{i} \overrightarrow{\mathrm{k}}_{1} & =R_{j}\left(\overrightarrow{\mathrm{k}}_{1}+\overrightarrow{\mathrm{K}}_{q_{i}}\right) \\
& =\overrightarrow{\mathrm{k}}_{1}+\overrightarrow{\mathrm{K}}_{q_{j}}+R_{j} \overrightarrow{\mathrm{K}}_{q_{i}}=\overrightarrow{\mathrm{k}}_{1}+\overrightarrow{\mathrm{K}}_{q_{p}} .
\end{aligned}
$$

This means that we may decompose $G$ in, for example, left cosets of the group $G_{\overrightarrow{\mathrm{k}}_{1}}$ as

$$
G=G_{\overrightarrow{\mathrm{k}}_{1}}+\left[P_{2} \mid \overrightarrow{\mathrm{p}}_{2}\right] G_{\overrightarrow{\mathrm{k}}_{1}}+\cdots\left[P_{s} \mid \overrightarrow{\mathrm{p}}_{s}\right] G_{\overrightarrow{\mathrm{k}}_{1}},
$$

where $\left[P_{i} \mid \vec{p}_{i}\right]$ denotes those operations in $G$, whose rotational elements rotate the $\vec{k}_{1}$ vector into a vector essentially different from $\vec{k}_{1}$; that is, relation (2.18) does not hold for these elements. We know from (2.17) that there exists just $s$ operations $\left[P_{i} \mid \overrightarrow{\mathrm{p}}_{i}\right]$ as $\left[P_{1} \mid \overrightarrow{\mathrm{p}}_{1}\right]=[\epsilon \mid \overrightarrow{0}]$. To make the notation clear, $\left[R_{i} \mid \overrightarrow{\mathrm{r}}_{i}\right], i=1, d$ denotes elements of the group of $\overrightarrow{\mathrm{k}}_{1}$ :

$$
R_{i} \overrightarrow{\mathrm{k}}_{1}=\overrightarrow{\mathrm{k}}_{1}+\overrightarrow{\mathrm{K}}_{a_{i}}
$$

$\left[P_{i} \mid \overrightarrow{\mathrm{p}}_{i}\right], i=1, s$ denotes elements characterized by

$$
P_{i} \overrightarrow{\mathrm{k}}_{1}=\overrightarrow{\mathrm{k}}_{i} \text {. }
$$

The set of vectors

$$
\overrightarrow{\mathrm{k}}_{1}, \overrightarrow{\mathrm{k}}_{2}, \ldots, \overrightarrow{\mathrm{k}}_{s}
$$

is also called the star of $\overrightarrow{\mathrm{k}}_{1}$, and the members of the star may be generated by the process

$$
P_{i} \overrightarrow{\mathrm{k}}_{1}=\overrightarrow{\mathrm{k}}_{i}, \quad i=1, \ldots, s .
$$

According to (2.19) we may characterize any element of $G$ as belonging to a particular coset of $G_{\overrightarrow{\mathrm{k}}_{1}}$. Thus, any element of the group $G,\left[S_{i} \mid \vec{t}_{i}\right]$, may be written

$$
\left[S_{i} \mid \overrightarrow{\mathrm{t}}_{i}\right]=\left[P_{m} \mid \overrightarrow{\mathrm{p}}_{m}\right]\left[R_{j} \mid \overrightarrow{\mathrm{r}}_{j}\right],
$$

where

$$
S_{i}=P_{m} R_{j}, \quad \overrightarrow{\mathrm{t}}_{i}=P_{m} \overrightarrow{\mathrm{r}}_{j}+\overrightarrow{\mathrm{p}}_{m}
$$

determines $m$ and $j$ unambiguously.

We have seen in (2.17) that our basis set $\left(\psi_{1}, \ldots, \psi_{n}\right)$ may be divided into sets, which are characterized by different transformation properties under the translation group. In order to emphasize this, we will from now on use two indices on the members of the basis set like $\psi_{\nu \mu}$. The first index $\nu$ indicates that the member transforms like $e^{i \overrightarrow{\mathrm{k}}_{\nu} \cdot \overrightarrow{\mathrm{T}}_{\alpha}}$ under $T$, while the second index indicates which member of the $\nu$ th set we consider. Thus we may write

$$
\left[\epsilon \mid \overrightarrow{\mathrm{T}}_{\alpha}\right] \psi_{1 \mu}=\exp \left(i \overrightarrow{\mathrm{k}}_{1} \cdot \overrightarrow{\mathrm{T}}_{\alpha}\right) \psi_{1 \mu}, \quad \mu=1, \ldots, d ;
$$

so for any element $\left[R_{i} \mid \overrightarrow{\mathrm{r}}_{i}\right]$ of $G_{\overrightarrow{\mathrm{k}}_{1}}$ we find

$$
\psi_{\mu}^{\prime}=\left[R_{i} \mid \vec{r}_{i}\right] \psi_{1 \mu}
$$

and

$$
\begin{aligned}
{\left[\epsilon \mid \overrightarrow{\mathrm{T}}_{\alpha}\right] \psi_{\mu}^{\prime} } & =\left[\epsilon \mid \overrightarrow{\mathrm{T}}_{\alpha}\right]\left[R_{i} \mid \overrightarrow{\mathrm{r}}_{i}\right] \psi_{1 \mu} \\
& =\left[R_{i} \mid \overrightarrow{\mathrm{r}}_{i}\right]\left[\epsilon \mid R_{i}^{-1} \overrightarrow{\mathrm{T}}_{\alpha}\right] \psi_{1 \mu} \\
& =\left[R_{i} \mid \overrightarrow{\mathrm{r}}_{i}\right] \exp \left[i\left(R_{i} \overrightarrow{\mathrm{k}}_{1}\right) \cdot \overrightarrow{\mathrm{T}}_{\alpha}\right] \psi_{1 \mu} \\
& =\exp \left(i \overrightarrow{\mathrm{k}}_{1} \cdot \overrightarrow{\mathrm{T}}_{\alpha}\right) \psi_{\mu}^{\prime} .
\end{aligned}
$$

The relation (2.21) holds of course for all $\mu$. This result may also be expressed by saying that the subspace which transforms according to $\overrightarrow{\mathrm{k}}_{1}$ under $T$, is invariant under $\mathrm{G}_{\overrightarrow{\mathrm{k}}_{1}}$. In this space any function, which transforms according to $\overrightarrow{\mathrm{k}}_{1}$, may be represented in the usual way:

$$
\psi=\left(\psi_{11}, \ldots, \psi_{1 d}\right)\left[\begin{array}{c}
s_{1} \\
\cdot \\
\cdot \\
\cdot \\
s_{d}
\end{array}\right]=\sum_{i} \psi_{1 i} s_{i} .
$$

Due to the invariance of the subspace, the function $\psi^{\prime}=\left[R_{i} \mid \overrightarrow{\mathrm{r}}_{i}\right] \psi$, therefore, also transforms according to $\overrightarrow{\mathrm{k}}_{1}$ under $T$. This means, that $\psi^{\prime}$ also may be represented in terms of the set $\left(\psi_{11}, \ldots, \psi_{1 d}\right)$ as

$$
\psi^{\prime}=\left(\psi_{11}, \ldots, \psi_{1 d}\right)\left[\begin{array}{c}
s_{1}^{\prime} \\
\cdot \\
\cdot \\
\cdot \\
s_{d}^{\prime}
\end{array}\right] .
$$

The fact that both $\psi$ and $\psi^{\prime}$ can be represented in the same space, means that the representation of $\left[R_{i} \mid \vec{r}_{i}\right]$ in this space is given in the usual way 


$$
\psi^{\prime}=\left[R_{i} \mid \overrightarrow{\mathrm{r}}_{i}\right] \psi=\left(\psi_{11}, \ldots, \psi_{1 d}\right) \underline{S}\left(\left[R_{i} \mid \overrightarrow{\mathrm{r}}_{i}\right]\right)\left[\begin{array}{c}
s_{1} \\
\cdot \\
\cdot \\
\cdot \\
s_{d}
\end{array}\right] .
$$

Note that the representation of $\left[R_{i} \mid \overrightarrow{\mathrm{r}}_{i}\right]$ is denoted by $S\left(\left[R_{i} \mid \vec{r}\right]\right)$. This is done in order to stress the fact that we have not yet found the wanted representation of $\left[R_{i} \mid \overrightarrow{\mathrm{r}}_{i}\right]$, which is called $D\left(\left[R_{i} \mid \overrightarrow{\mathrm{r}}_{i}\right]\right) \times$ $S\left(\left[R_{i} \mid \overrightarrow{\mathrm{r}}_{i}\right]\right)$ is thus a $d \times d$ matrix, while $\bar{D}\left(\left[R_{i} \mid \overrightarrow{\mathrm{r}}_{i}\right]\right)$ is a $n \times n$ matrix, so we have only found the representation of $\left[R_{i} \mid \overrightarrow{\mathrm{r}}_{i}\right]$ in the space spanned by $\left(\psi_{11}, \ldots, \psi_{1 d}\right)$. This is an important result which we are going to make use of in a moment.

Let us next investigate, how the function $\psi_{\mu}^{\prime}$ defined according to

$$
\psi_{\mu}^{\prime}=\left[P_{i} \mid \vec{p}_{i}\right] \psi_{1 \mu}, \quad \mu=1, \ldots, d
$$

transforms under $T$. We find

$$
\begin{aligned}
{\left[\epsilon \mid \overrightarrow{\mathrm{T}}_{\alpha}\right] \psi_{\mu}^{\prime} } & =\left[\epsilon \mid \overrightarrow{\mathrm{T}}_{\alpha}\right]\left[P_{i} \mid \overrightarrow{\mathrm{p}}_{i}\right] \psi_{1 \mu} \\
& =\left[P_{i} \mid \overrightarrow{\mathrm{p}}_{i}\right]\left[\epsilon \mid P_{i}^{-1} \overrightarrow{\mathrm{T}}_{\alpha}\right] \psi_{1 \mu} \\
& =\left[P_{i} \mid \overrightarrow{\mathrm{p}}_{i}\right] \times \exp \left[i \overrightarrow{\mathrm{k}}_{1} \cdot\left(P_{i}^{-1} \overrightarrow{\mathrm{T}}_{\alpha}\right)\right] \psi_{1 \mu} \\
& =\exp \left[i\left(P_{i} \overrightarrow{\mathrm{k}}_{1}\right) \cdot \overrightarrow{\mathrm{T}}_{\alpha}\right]\left[P_{i} \mid \overrightarrow{\mathrm{p}}_{i}\right] \psi_{1 \mu} \\
& =\exp \left[i\left(P_{i} \overrightarrow{\mathrm{k}}_{1}\right) \cdot \overrightarrow{\mathrm{T}}_{\alpha}\right] \psi_{\mu}^{\prime},
\end{aligned}
$$

that is, in (2.23) we generate a "function" which transforms according to $\overrightarrow{\mathrm{k}}_{i}$ under $T$. In contrary to the result found above, we see that $\psi^{\prime}$ cannot be represented in the space of $\left(\psi_{11}, \ldots, \psi_{1 d}\right)$.

It is now remembered, that the basis "functions" in the $i$ th block of our basis set $\left(\psi_{1}, \ldots, \psi_{n}\right)$ just transform according to $\overrightarrow{\mathrm{k}}_{i}$ under $T$. Therefore we may generate the basis functions in the $i$ th block from the basis in the first block by application of the operation $\left[P_{i} \mid \overrightarrow{\mathrm{p}}_{i}\right]$ :

$\psi_{i \mu}=\left[P_{i} \mid \overrightarrow{\mathrm{p}}_{i}\right] \psi_{1 \mu}, \quad i=1, \ldots, s, \quad \mu=1, \ldots, d$.

Let us assume that the members in our basis set $\left(\psi_{1}, \ldots, \psi_{n}\right)$ satisfy this relation.

Finally, we are now in the position to deduce the form of the representation matrices of any operation $[S \mid \vec{t}]$ in the space of $\left(\psi_{1}, \ldots, \psi_{n}\right)$. Let $[S \mid \overrightarrow{\mathrm{t}}]$ work on $\psi_{l \mu}$ and we find

$$
\begin{aligned}
{[S \mid \overrightarrow{\mathrm{t}}] \psi_{l \mu} } & =[S \mid \overrightarrow{\mathrm{t}}]\left[P_{l} \mid \overrightarrow{\mathrm{p}}_{l}\right] \psi_{1 \mu} \\
& =\left[P_{m} \mid \overrightarrow{\mathrm{p}}_{m}\right]\left[R_{j} \mid \overrightarrow{\mathrm{r}}_{j}\right] \psi_{1 \mu},
\end{aligned}
$$

where we have used (2.24) and the fact that any element of $G,[S \mid \vec{t}]\left[P_{l} \mid \vec{p}_{l}\right]$, belongs to a particular coset of $G_{\overrightarrow{\mathrm{k}}_{1}}$, here the $m$ th $[(2.20)]$.
From (2.22) we now know that

$$
\begin{aligned}
{\left[R_{j} \mid \overrightarrow{\mathrm{r}}_{j}\right] \psi_{1 \mu} } & =\left(\psi_{11}, \ldots, \psi_{1 d}\right) \underline{S}\left(\left[R_{j} \mid \overrightarrow{\mathrm{r}}_{j}\right]\right)\left[\begin{array}{c}
0 \\
\vdots \\
1 \\
\vdots \\
0
\end{array}\right] \\
& =\sum_{i} \psi_{1 i} S_{i \mu}\left(\left[R_{j} \mid \overrightarrow{\mathrm{r}}_{j}\right]\right),
\end{aligned}
$$

so

$$
\begin{aligned}
{[S \mid \overrightarrow{\mathrm{t}}] \psi_{l \mu}=} & {\left[P_{m} \mid \overrightarrow{\mathrm{p}}_{m}\right] \sum_{i} \psi_{1 i} S_{i \mu}\left(\left[R_{j} \mid \overrightarrow{\mathrm{t}}_{j}\right]\right) } \\
= & \sum_{i} \psi_{m i} S_{i \mu}\left(R_{j} \mid \overrightarrow{\mathrm{r}}_{j}\right), \\
& \mu=1, \ldots, d, \quad i=1, \ldots, d,
\end{aligned}
$$

where we have used (2.24).

To make the results in Eq. (2 .26) clear, let us write $\psi^{\prime}=[S \mid \vec{t}] \psi_{l \mu}$ in the space of $\left(\psi_{1}, \ldots, \psi_{n}\right)$ :

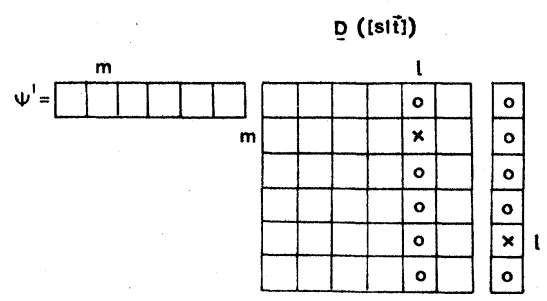

where

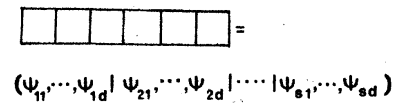

shows the block structure of the basis, and the component matrix

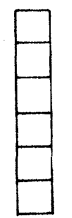

shows the same block structure. The representation matrix for the symmetry operation therefore also has a block structure as shown, where the dimension of each block is $d \times d$. It is of course of no significance that we use six blocks in (2.27). The situation in (2.26) does now correspond to the case, where just the $\mu$ th element in the $l$ th block of the component matrix is one. The rest of the matrix elements are zero. This is shown in (2 .27) by a cross and by zeros. Then according to (2.26), all elements in the $l$ th column of blocks in $D([S \mid \vec{t}])$ are zero, besides in the $m$ th row of blocks of $\underline{D}([S \mid \vec{t}])$, where elements different from 
zero occur. This block is also shown with a cross, while the rest of the blocks are zero. As we let $\mu$ go from 1 to $d$, we see that the $d \times d$ matrix at the place of the cross in $D([S \mid \vec{t}])$ just is the ma$\operatorname{trix} S\left(\left[R_{j} \mid \overrightarrow{\mathrm{r}}_{j}\right]\right)$. In this way we may deduce the structure of $D([S \mid \overrightarrow{\mathrm{t}}])$ letting $l=1, \ldots, s$.

Irreducible representations of $S\left(\left[R_{i} \mid \vec{r}_{i}\right]\right)$. In order to find the irreducible representations of a space group associated with a reciprocal vector $\overrightarrow{\mathrm{k}}_{1}$, we have to find the irreducible representations of $S\left(\left[R_{i} \mid \overrightarrow{\mathrm{r}}_{i}\right]\right)$ in the space of $\left(\psi_{11}, \ldots, \psi_{1 d}\right)$. We will pursue this topic here. The rotational elements $S$ of a space group, whose elements are $[S \mid \vec{t}]$, form one of the 32 point groups $G^{0}$. Also, the rotational elements of $G \overrightarrow{\mathrm{k}}_{1}$ form a point group, which is a subgroup of $G^{0}$. We have seen that, as $\vec{k}_{1}$ wanders over the interior and the surface of the Brillouin zone, we may obtain all irreducible representations of $G$ by finding the irreducible representations of the group of the $\vec{k}_{1}$ vector in question in the space of $\left(\psi_{11}, \ldots, \psi_{1 d}\right)$.

Let us first study points in the interior of the Brillouin zone. For an element $\left[R_{i} \mid \vec{r}_{i}\right]$ of the group of $\overrightarrow{\mathrm{k}}_{1}$, we know in general that $R_{i} \overrightarrow{\mathrm{k}}_{1}=\overrightarrow{\mathrm{k}}_{1}+\overrightarrow{\mathrm{K}}_{q}$. In this case, where we consider vectors in the interior of the Brillouin zone, we have $\overrightarrow{\mathrm{K}}_{a_{i}}=0$, so

$$
R_{i} \overrightarrow{\mathrm{k}}_{1}=\overrightarrow{\mathrm{k}}_{1} \text {. }
$$

Any element of $G_{\vec{k}_{1}},\left[R_{i} \mid \overrightarrow{\mathrm{r}}_{i}\right]$, may be written

$$
\left[R_{i} \mid \overrightarrow{\mathrm{r}}_{i}\right]=\left[\epsilon \mid \overrightarrow{\mathrm{T}}_{r_{i}}\right]\left[R_{i} \mid \overrightarrow{\mathrm{r}}_{R_{i}}\right] \text {, }
$$

where $\overrightarrow{\mathrm{r}}_{R_{i}}$ is a fractional (nonprimitive) translation vector (occurs for at least one element if $G_{\overrightarrow{\mathrm{k}}_{1}}$ is one of the 157 nonsymmorphic groups) or zero (all elements in the 73 symmorphic groups), while $\overrightarrow{\mathrm{T}}_{r_{i}}$ is a primitive translation. Therefore we may rewrite (2.29) as follows:

$$
\begin{aligned}
\underline{S}\left(\left[R_{i} \mid \overrightarrow{\mathrm{r}}_{i}\right]\right) & =\exp \left(i \overrightarrow{\mathrm{k}}_{1} \circ \overrightarrow{\mathrm{T}}_{r_{i}}\right) \underline{S}\left(\left[R_{i} \mid \overrightarrow{\mathrm{r}}_{R_{i}}\right]\right) \\
& =\exp \left[i \overrightarrow{\mathrm{k}}_{1} \circ\left(\overrightarrow{\mathrm{T}}_{r_{i}}+\overrightarrow{\mathrm{r}}_{R_{i}}\right)\right] \underline{\Gamma}\left(\left[R_{i}\right]\right),
\end{aligned}
$$

which defines $\underline{\Gamma}\left(\left[R_{i}\right]\right)$. The representation of $\left[R_{i} \mid \overrightarrow{\mathrm{r}}_{R_{i}}\right]$ in the space of $\left(\psi_{11}, \ldots, \psi_{1 d}\right)$ is thus given by

$$
\underline{S}\left(\left[R_{i} \mid \overrightarrow{\mathrm{r}}_{R_{i}}\right]\right)=\exp \left(i \overrightarrow{\mathrm{k}}_{1} \cdot \overrightarrow{\mathrm{r}}_{R_{i}}\right) \underline{\Gamma}\left(\left[R_{i}\right]\right) .
$$

Note that $\overrightarrow{\mathrm{r}}_{R_{i}}$ is a nonprimitive translational vector or zero, and $\Gamma\left(\left[R_{i}\right]\right)$ depends only on the rotational part of the operation.

We will show that the matrix $\underline{\Gamma}\left(\left[R_{i}\right]\right)$ defined above simply is the irreducible representation matrix of the point group $G_{\overrightarrow{\mathrm{k}}_{1}}^{0}$. To see this we have to prove that for any two elements of $G_{\mathrm{k}_{1}}^{s_{1}}$ we have

$$
\left[R_{i} \mid \overrightarrow{\mathrm{r}}_{R_{i}}\right]\left[R_{j} \mid \overrightarrow{\mathrm{r}}_{R_{j}}\right]=\left[R_{i} R_{j} \mid R_{i} \overrightarrow{\mathrm{r}}_{R_{j}}+\overrightarrow{\mathrm{r}}_{R_{i}}\right] \text {. }
$$

Inserting our representation of the operations, we find

$$
\begin{aligned}
{\left[R_{i} \mid \overrightarrow{\mathrm{r}}_{R_{i}}\right]\left[R_{j} \mid \overrightarrow{\mathrm{r}}_{R_{j}}\right]=} & \exp \left(i \overrightarrow{\mathrm{k}}_{1} \cdot \overrightarrow{\mathrm{r}}_{R_{i}}\right) \exp \left(i \overrightarrow{\mathrm{k}}_{1} \cdot \overrightarrow{\mathrm{r}}_{R_{j}}\right) \\
& \times \underline{\Gamma}\left(\left[R_{i}\right]\right) \underline{\Gamma}\left(\left[R_{j}\right]\right) \\
& =\exp \left[i \overrightarrow{\mathrm{k}}_{1} \circ\left(\overrightarrow{\mathrm{r}}_{R_{i}}+\overrightarrow{\mathrm{r}}_{R_{j}}\right)\right] \underline{\Gamma}\left(\left[R_{i}\right]\right) \underline{\Gamma}\left(\left[R_{j}\right]\right)
\end{aligned}
$$

and

$$
\begin{aligned}
{\left[R_{i} R_{j} \mid R_{i} \overrightarrow{\mathrm{r}}_{R_{j}}\right.} & \left.+\overrightarrow{\mathrm{r}}_{R_{i}}\right] \\
& =\exp \left[i \overrightarrow{\mathrm{k}}_{1} \circ\left(R_{i} \overrightarrow{\mathrm{r}}_{R_{j}}+\overrightarrow{\mathrm{r}}_{R_{i}}\right)\right] \underline{\Gamma}\left(\left[R_{i} R_{j}\right]\right) \\
& =\exp \left\{i\left[\left(R_{i}^{-1} \overrightarrow{\mathrm{k}}_{1}\right) \circ \overrightarrow{\mathrm{r}}_{R_{j}}+\overrightarrow{\mathrm{k}}_{1} \circ \overrightarrow{\mathrm{r}}_{R_{i}}\right]\right\} \underline{\Gamma}\left(\left[R_{i} R_{j}\right]\right) \\
& =\exp \left[i \overrightarrow{\mathrm{k}}_{1} \cdot\left(\overrightarrow{\mathrm{r}}_{R_{j}}+\overrightarrow{\mathrm{r}}_{R_{i}}\right)\right] \underline{\Gamma}\left(\left[R_{i} R_{j}\right]\right), \quad(2.32)
\end{aligned}
$$

where we have used $(2.28)$. We see that the righthand side of Eqs. (2.31) and (2.32) are identical if and only if

$$
\underline{\Gamma}\left(\left[R_{i}\right]\right) \underline{\Gamma}\left(\left[R_{j}\right]\right)=\underline{\Gamma}\left(\left[R_{i} R_{j}\right]\right),
$$

and we know from ordinary point-group theory that this is the case if $\Gamma\left(\left[R_{i}\right]\right)$ and $\Gamma\left(\left[R_{j}\right]\right)$ are the irreducible representations of the point group $G_{\overrightarrow{\mathrm{k}}_{1}}^{0}$.

To conclude, we have seen that

$$
\underline{S}\left(\left[R_{i} \mid \overrightarrow{\mathrm{r}}_{R_{i}}\right]\right)=\exp \left(i \overrightarrow{\mathrm{k}}_{1} \cdot \overrightarrow{\mathrm{r}}_{R_{i}}\right) \underline{\Gamma}\left(\left[R_{i}\right]\right),
$$

where $\Gamma\left(\left[R_{i}\right]\right)$ is the irreducible representation of the point group $G_{\overrightarrow{\mathrm{k}}_{1}}^{0}$, constitutes a representation of $\left[R_{i} \mid \overrightarrow{\mathrm{r}}_{\mathrm{R}_{i}}\right]$ in the space of $\left(\psi_{11}, \ldots, \psi_{1 d}\right)$ for both symmorphic and nonsymmorphic groups, if $\overrightarrow{\mathrm{k}}_{1}$ is any vector inside the Brillouin zone. From this it is easy to construct the irreducible representations for all elements in the space group, as we have seen, (2.27).

$\overrightarrow{\mathrm{k}}_{1}$ on the surfaces of the Brillouin zone. The equation corresponding to $(2.28)$ now has the form $R_{i} \overrightarrow{\mathrm{k}}_{1}=\overrightarrow{\mathrm{k}}_{1}+\overrightarrow{\mathrm{K}}_{q_{i}}$, where $\overrightarrow{\mathrm{K}}_{q_{i}}$ is a primitive vector in reciprocal space different from zero. The equations analogous to (2.31) and (2.32) are

$$
\begin{aligned}
& {\left[R_{i} \mid \overrightarrow{\mathrm{r}}_{R_{i}}\right]\left[R_{j} \mid \overrightarrow{\mathrm{r}}_{R_{j}}\right] } \\
& \quad=\exp \left[i \overrightarrow{\mathrm{k}}_{1} \circ\left(\overrightarrow{\mathrm{r}}_{R_{i}}+\overrightarrow{\mathrm{r}}_{R_{j}}\right)\right] \underline{\Gamma}\left(\left[R_{i}\right]\right) \underline{\Gamma}\left(\left[R_{j}\right]\right)
\end{aligned}
$$

and

$$
\begin{aligned}
{\left[R_{i} R_{j} \mid\right.} & \left.R_{i} \overrightarrow{\mathrm{r}}_{R_{j}}+\overrightarrow{\mathrm{r}}_{R_{i}}\right] \\
& =\exp \left[i \overrightarrow{\mathrm{k}}_{1} \circ\left(R_{i} \overrightarrow{\mathrm{r}}_{R_{j}}+\overrightarrow{\mathrm{r}}_{R_{i}}\right)\right] \underline{\Gamma\left(\left[R_{i} R_{j}\right]\right)} \\
& =\exp \left\{i\left[\left(R_{i}^{-1} \overrightarrow{\mathrm{k}}_{1}\right) \circ \overrightarrow{\mathrm{r}}_{R_{j}}+\overrightarrow{\mathrm{k}}_{1} \circ \overrightarrow{\mathrm{r}}_{R_{i}}\right]\right\} \underline{\Gamma}\left(\left[R_{i} R_{j}\right]\right) \\
& =\exp \left(i \cdot \overrightarrow{\mathrm{K}}_{q^{\prime}} \cdot \overrightarrow{\mathrm{r}}_{R_{j}}\right) \exp \left[i \overrightarrow{\mathrm{k}}_{1} \cdot\left(\overrightarrow{\mathrm{r}}_{R_{j}}+\overrightarrow{\mathrm{r}}_{R_{i}}\right)\right] \underline{\Gamma}\left(\left[R_{i} R_{j}\right]\right),
\end{aligned}
$$

where $\overrightarrow{\mathrm{K}}_{q^{\prime}}$ is different from zero and given by

$$
R_{i}^{-1} \overrightarrow{\mathrm{k}}_{1}=\overrightarrow{\mathrm{k}}_{1}+\overrightarrow{\mathrm{K}}_{q^{\prime}} .
$$

This result is in general different from the result in the preceding case. The multiplication rules for the matrices $\underset{\Gamma}{\Gamma}\left(\left[R_{i}\right]\right)$ are here found to be 


$$
\underline{\Gamma}\left(\left[R_{i}\right]\right) \underline{\Gamma}\left(\left[R_{j}\right]\right)=\exp \left(i \overrightarrow{\mathrm{K}}_{q^{\prime}} \cdot \overrightarrow{\mathrm{r}}_{R_{j}}\right) \underline{\Gamma}\left(\left[R_{i} R_{j}\right]\right),
$$

or from (2.35)

$$
\begin{aligned}
\underline{\Gamma}\left(\left[R_{i}\right]\right) & \underline{\Gamma}\left(\left[R_{j}\right]\right) \\
& =\exp \left[i \overrightarrow{\mathrm{k}}_{1} \cdot\left(R_{i} \overrightarrow{\mathrm{r}}_{R_{j}}-\overrightarrow{\mathrm{r}}_{R_{j}}\right)\right] \underline{\Gamma}\left(\left[R_{i} R_{j}\right]\right) .
\end{aligned}
$$

This shows, that $\Gamma\left(\left[R_{i}\right]\right)$ is not an irreducible representation matrix in the ordinary sense, due to the exponential factors. The matrices $\Gamma\left(\left[R_{i}\right]\right)$ are said to form a multiplier representation of the point group $G_{\overrightarrow{\mathrm{k}}_{1}}^{0}$. In the special case, where we consider a symmorphic group all $\vec{r}_{R_{j}}=0$ and therefore all exponential factors will be equal to 1 , which means that $\Gamma\left(\left[R_{i}\right]\right)$ in this case is an ordinary representation of the point group. Also, if $\overrightarrow{\mathrm{k}}_{1}$ is in the interior of the Brillouin zone, we have

$$
\exp \left[i \overrightarrow{\mathrm{k}}_{1} \cdot\left(R_{i} \overrightarrow{\mathrm{r}}_{R_{j}}-\overrightarrow{\mathrm{r}}_{R_{j}}\right)\right]=\exp \left[i \overrightarrow{\mathrm{k}}_{1} \cdot\left(\overrightarrow{\mathrm{r}}_{R_{j}}-\overrightarrow{\mathrm{r}}_{R_{j}}\right)\right]=1,
$$

which again means that the factor system is equal to 1 , so $\underline{\Gamma}\left(\left[R_{i}\right]\right)$ again is an ordinary representation. Only in the case where we consider $\overrightarrow{\mathrm{k}}_{1}$ on the surface of the Brillouin zone, and consider nonsymmorphic groups, do we have to know the multiplier representations of the point group $G_{\overrightarrow{\mathrm{k}}}^{0}$. We may say that the multiplication scheme in (2.36) includes all possibilities.

Summary. The dimension of the representation matrices depends on the vector $\overrightarrow{\mathrm{k}}_{1}$ considered. If the star of $\overrightarrow{\mathrm{k}}_{1}$ includes $s \overrightarrow{\mathrm{k}}$ vectors, the matrices are $s \times s$ block matrices (2.27). The structure of the matrices is such, that only one block in each column and row of blocks is different from the null matrix. The blocks, which are different from the null matrix, are the $(m, l)$ blocks, where $m$ and $l$ are given by (2.25):

$$
[S \mid \overrightarrow{\mathrm{t}}]\left[P_{l} \mid \overrightarrow{\mathrm{p}}_{l}\right]=\left[P_{m} \mid \overrightarrow{\mathrm{p}}_{m}\right]\left[R_{j} \mid \overrightarrow{\mathrm{r}}_{j}\right],
$$

when we consider the representation of the $[S \mid \vec{t}]$ operation. The nonzero blocks are given by the matrices

$$
\underline{S}\left(\left[R_{j} \mid \overrightarrow{\mathrm{r}}_{j}\right]\right)=\exp \left[i \overrightarrow{\mathrm{k}}_{1} \cdot\left(\overrightarrow{\mathrm{T}}_{j}+\overrightarrow{\mathrm{r}}_{R_{j}}\right)\right] \underline{\Gamma}\left(\left[R_{j}\right]\right),
$$

where $\overrightarrow{\mathrm{T}}_{j}$ is zero or a primitive translation given by $\overrightarrow{\mathrm{r}}_{j}=\overrightarrow{\mathrm{T}}_{j}+\overrightarrow{\mathrm{r}}_{R_{j}} \cdot \overrightarrow{\mathrm{r}}_{R_{j}}$ is a nonprimitive translation or zero. The $\Gamma\left(\left[R_{j}\right]\right)$ matrices are the multiplier representations of the point group $G_{\overrightarrow{\mathrm{k}}_{1}}^{0}$ with the multiplication rule

$$
\begin{aligned}
\underline{\Gamma}\left(\left[R_{i}\right]\right) & \underline{\Gamma}\left(\left[R_{j}\right]\right) \\
& =\exp \left[i \overrightarrow{\mathrm{k}}_{1} \cdot\left(R_{i} \overrightarrow{\mathrm{r}}_{R_{j}}-\overrightarrow{\mathrm{r}}_{R_{j}}\right)\right] \underline{\Gamma}\left(\left[R_{i} R_{j}\right]\right) .
\end{aligned}
$$

In certain cases the $\Gamma\left(\left[R_{i}\right]\right)$ matrices reduce to ordinary point-group representation matrices. The different cases are (i) if $\vec{k}_{1}$ is an interior vector of the Brillouin zone, the $\Gamma\left(\left[R_{i}\right]\right)$ matrices are ordinary point-group representations; (ii) if we consider a symmorphic group, the $\underline{\Gamma}\left(\left[R_{i}\right]\right)$ matrices are ordinary point-group representations; (iii) if $\vec{k}_{1}$ is on the surface of the Brillouin zone, and we consider a nonsymmorphic group, the $\Gamma\left(\left[R_{i}\right]\right)$ matrices are multiplier representations of the group. The basic assumption for the representations of $[S \mid \vec{t}]$ as given above was that we considered a basis set $\left(\psi_{11}, \ldots, \psi_{1 d}\right)$, which spanned the subspace and transformed according to $\overrightarrow{\mathrm{k}}_{1}$ under $T$. There will be such a set for each of the irreducible representations found in the point group $G G_{\mathrm{k}_{1}}^{0}$. The complete basis set, which spans the representation of $G$ was found from (2.24) by

$$
\left[P_{i} \mid \overrightarrow{\mathrm{p}}_{i}\right] \psi_{1 \mu}=\psi_{i \mu}, \quad i=1, \ldots, s, \quad \mu=1, \ldots, d,
$$

where the $\left[P_{i} \mid \vec{p}_{i}\right]$ are the operations used to produce the members of the star of $\vec{k}_{1}$. If the dimension of the representation is $n$, then it was found that $n=s d$.

Multiplier representations. We have seen that only for $\vec{k}$ vectors on the surface of the Brillouin zone and for nonsymmorphic groups, the multiplier representations of the point groups of the $\vec{k}$ vector. are different from ordinary representations.

In Kovalev's book ${ }^{7}$ one may find the multiplier representations for various point groups $G_{\overrightarrow{\mathrm{k}}_{1}}^{0}$ associated with a space group $G$. All space groups are covered in that way. In order to be able to check the representations for misprints and other possible errors, it is useful to know how to construct these representations.

To do so one has just to construct the multiplication table of the rotational elements in $G_{\overrightarrow{\mathrm{k}}_{1}}^{0}$ according to (2.39). From this it is possible to obtain the multiplier representations. The method is demonstrated later in an example (see Sec. III B). The representations may also be obtained by the computer code of Worlton and Warren. ${ }^{12}$

In order to avoid future confusion it is stressed, that the equation corresponding to (2.39) in Kovalev's book ${ }^{7}$ has the form

$$
\begin{aligned}
& \underline{\Gamma}\left(\left[R_{i}\right]\right) \underline{\Gamma}\left(\left[R_{j}\right]\right) \\
& =\exp \left[-i \overrightarrow{\mathrm{k}}_{1} \cdot\left(R_{i} \overrightarrow{\mathrm{r}}_{R_{j}}-\overrightarrow{\mathrm{r}}_{R_{j}}\right)\right] \Gamma\left(\left[R_{i} R_{j}\right]\right) .
\end{aligned}
$$

The minus sign in the exponential is, of course, a reflection of the fact that he has defined his $\underline{\Gamma}\left(\left[R_{i}\right]\right)$ matrices according to

$$
\underline{S}\left(\left[R_{i} \mid \overrightarrow{\mathrm{r}}_{R_{i}}\right]\right)=\exp \left(-i \overrightarrow{\mathrm{k}}_{1} \cdot \overrightarrow{\mathrm{r}}_{R_{i}}\right) \underline{\Gamma}\left(\left[R_{i}\right]\right),
$$

where we have used (2.38):

$$
\underline{S}\left(\left[R_{i} \mid \overrightarrow{\mathrm{r}}_{R_{i}}\right]\right)=\exp \left(i \overrightarrow{\mathrm{k}}_{1} \cdot \overrightarrow{\mathrm{r}}_{R_{i}}\right) \Gamma\left(\left[R_{i}\right]\right) .
$$

When we consider the group of $\overrightarrow{\mathrm{k}}_{1}$, then it is equivalent in the formalism of Kovalev to consider the group of $-\vec{k}_{1}$. These differences are of no practical importance, as the groups of $\vec{k}_{1}$ and $-\vec{k}_{1}$ include the same set of symmetry operations. 
Generation of a basis set for the space group $G$. In order to find a basis set, which spans the group $G$, we have seen that for any $\overrightarrow{\mathrm{k}}$ vector $\overrightarrow{\mathrm{k}}_{1}$, we have to find a set $\psi_{11}, \ldots, \psi_{1 d}$ for each of the irreducible representations found in the point group $G_{\mathrm{k}_{1}}^{0}$. The complete set is then generated using $(2.24)$ for every set of $\psi_{11}, \ldots, \psi_{1 d}$. To find the set $\psi_{11}, \ldots, \psi_{1 d}$ we use the projection-operator technique. The projection operator is defined according to $\mathrm{t}^{9,10,11,13}$

$$
\hat{p}_{r, r}^{(\lambda)}=\frac{l_{\lambda}}{g} \sum_{i=1}^{g} \chi_{r r}^{(\lambda) *}\left(R_{i}\right)\left[R_{i} \mid \vec{r}_{R_{i}}\right],
$$

where $l_{\lambda}$ is the dimension of the $\lambda$ th irreducible representation, $g$ is the number of operations in $G_{\overrightarrow{\mathrm{k}}_{1}}^{0}, \chi_{r r}^{(\lambda) *}\left(R_{i}\right)$ the complex conjugate of the $(r, r)$ th element in the ir reducible representation matrix for the $\lambda$ th irreducible representation, and $\left[R_{i} \mid \overrightarrow{\mathrm{r}}_{R_{j}}\right]$ is the $i$ th operation in the group of $\overrightarrow{\mathrm{k}}_{1}, G_{\mathrm{k}_{1}}^{0}$. $\chi_{r r}^{(\lambda)}\left(R_{i}\right)$ is found in Kovalev's tables of the multiplier representations of the point group $G_{\overrightarrow{\mathrm{k}}_{1}}^{0}$, and $\left[R_{i} \mid \overrightarrow{\mathbf{r}}_{R_{i}}\right]$ is represented by

$$
\underline{M}\left(\left[R_{i} \mid \overrightarrow{\mathrm{r}}_{R_{i}}\right]\right)=\exp \left(i \overrightarrow{\mathrm{k}}_{1} \cdot \overrightarrow{\mathrm{r}}_{R_{i}}\right) \underline{L}\left(\left[R_{i}\right]\right) .
$$

In order to stress the fact that the representation of $\left[R_{i} \mid \vec{r}_{R_{i}}\right]$, in general, is given in a basis set different from the ones we have considered until now, other letters ( $M$ and $L$ ) have been used to designate the representation matrices. In which basis do we now represent $\left[R_{i} \mid \overrightarrow{\mathrm{r}}_{R_{i}}\right]$ ? That depends completely on the problem. In the case of lattice vibrations, where we want to project out a set of displacement patterns (symmetry coordinates), which transform under $G_{\mathrm{k}_{1}}$ as prescribed by the matrices of the irreducible representations of $G_{\overrightarrow{\mathrm{k}}_{1}}$, we usually express the symmetry coordinates in terms of Cartesian displacements of the atoms in the unit cell.

Therefore, in this case, the answer to the question raised above is that $\left[R_{i} \mid \overrightarrow{\mathrm{r}}_{R_{i}}\right]$ is represented in the basis used to describe the displacements of the atoms in the unit cell. Usually these are simple local Cartesian unit vectors with origins at the equilibrium positions of the atoms in the unit cell, and oriented in the same way. If there are $n$ atoms in the unit cell, our basis for $\left[R_{i} \mid \overrightarrow{\mathrm{r}}_{R_{i}}\right]$ will be the
$3 n$ Cartesian unit vectors, and the dimension of the representations is $3 n \times 3 n$.

Letting the projection operators work on any displacement pattern given in this space, we will generate symmetry coordinates, which span the $\lambda$ th irreducible representation and transform as the $r$ th row in the ir reducible-representation matrices. Having this displacement pattern, we may let the projection operator

$$
\hat{p}_{s, r}^{(\lambda)}
$$

work on this displacement pattern in order to obtain its partners, which transform according to the $s$ th row of the irreducible-representation matrix. We will not give the proof of these important relations here, just note that the relations are all consequences of the "great orthogonality theorem," which is also valid for multiplier representations.

\section{EXAMPLE}

Let us try to apply the theory developed above on a specific example. We consider in the following crystalline trigonal selenium. The crystal class is the $D_{3}^{6}$ group, and the symmetry operations are taken from Kovalev's book and listed in Table I. The structure of Se is shown in Fig. 1.

Kovalev calls the rotational part of a symmetry operation $h_{i}$. In the following we will use $S_{i}$, with the same $i$ as Kovalev, to facilitate the identification of the operation. The section is divided into three parts. In Sec. III A we give the representations of $G$ for various $\overrightarrow{\mathrm{k}}$ vectors, and in Sec. III B we show how to generate the multiplier representations of the point groups, so one may check the results in Kovalev's tables for misprints and other possible errors. In Sec. IIIC we illustrate the generation of symmetry coordinates in a lattice-dynamical problem on selenium.

\section{A. Generation of the irreducible representations of the $D_{3}^{6}$ group}

For the analysis, it will be convenient to have a multiplication table for the rotational part of the

\begin{tabular}{|c|c|c|}
\hline \multicolumn{3}{|c|}{ Kovalev, $D_{3}^{6}$} \\
\hline $\begin{array}{l}\left(h_{1} \mid \overrightarrow{0}\right): \\
\left(h_{3} \mid \frac{1}{3} \vec{a}_{3}\right): \\
\left(h_{5} \mid \frac{2}{3} \vec{a}_{3}\right): \\
\left(h_{7} \mid \overrightarrow{0}\right): \\
\left(h_{9} \mid \frac{1}{3} \vec{a}_{3}\right): \\
\left(h_{11} \mid \frac{2}{3} \vec{a}_{3}\right): \\
\left(h_{1} \mid \overrightarrow{\mathrm{T}}_{\alpha}\right):\end{array}$ & $\left.\begin{array}{l}\text { identity } \\
120^{\circ} \text { rotation around }(0,0,1) \\
240^{\circ} \text { rotation around }(0,0,1) \\
180^{\circ} \text { rotation around }(0,1,0) \\
180^{\circ} \text { rotation around }(1,0,0) \\
180^{\circ} \text { rotation around }(1,1,0) \\
\text { primitive translation } \overrightarrow{\mathrm{T}}_{\alpha}=\alpha_{1} \overrightarrow{\mathrm{a}}_{1}+\end{array}\right\}$ & $\begin{array}{l}\text { All components are } \\
\text { given in terms of } \\
\text { the skew coordinate } \\
\text { system }\left(\overrightarrow{\mathrm{a}}_{1}, \overrightarrow{\mathrm{a}}_{2}, \overrightarrow{\mathrm{a}}_{3}\right) \text {. } \\
\text { See Fig. } 1 . \\
\alpha_{2} \overrightarrow{\mathrm{a}}_{2}+\alpha_{3} \overrightarrow{\mathrm{a}}_{3}\end{array}$ \\
\hline
\end{tabular}
operations as shown in Table II.

TABLE I. Symmetry operations for the $D_{3}^{6}$ space group from Kovalev (Ref. 7). 


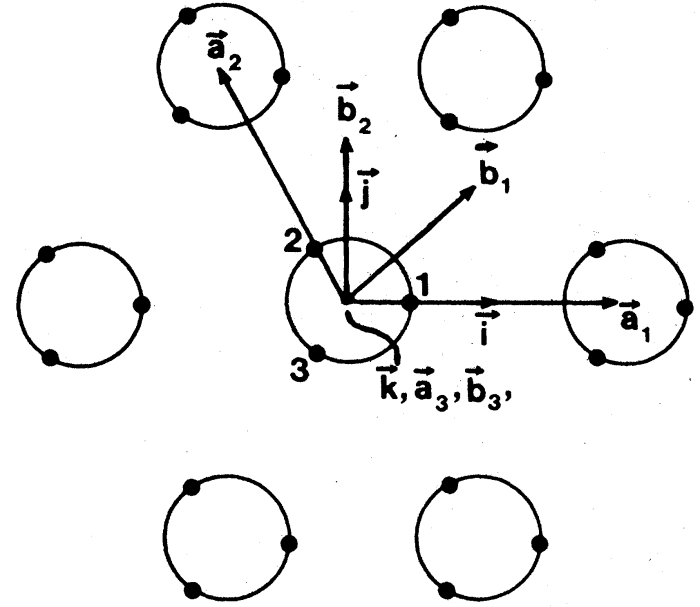

FIG. 1. Structure of trigonal selenium. The atoms are arranged in spiral chains, which are arranged in a hexagonal pattern. The lattice basis vectors $\left(\vec{a}_{1}, \vec{a}_{2}\right.$, $\left.\vec{a}_{3}\right)$, the Cartesian coordinate system $(\vec{i}, \vec{j}, \vec{k})$, and the reciprocal-lattice basis vectors $\left(\vec{b}_{1}, \vec{b}_{2}, \vec{b}_{3}\right)$ are also shown. The angle between $\vec{a}_{1}$ and $\vec{a}_{2}$ is $120^{\circ}$, between $\vec{a}_{1}$ and $\vec{a}_{3}$ is $90^{\circ}$, and between $\vec{a}_{2}$ and $\vec{a}_{3}$ is $90^{\circ}$.

Let us consider the wave vector $\vec{k}_{1}$ given by

$$
\vec{k}_{1}=\left(\vec{a}_{1}, \vec{a}_{2}, \vec{a}_{3}\right)\left[\begin{array}{l}
0 \\
0 \\
\mu
\end{array}\right]
$$

for various values of $\mu$.

$$
\text { 1. } \mu=0
$$

The group of the wave vector $G_{\overrightarrow{\mathrm{k}}_{1}}=G$ for $\overrightarrow{\mathrm{k}}_{1}=0$. Also, as we consider a point inside the Brillouin zone the $\Gamma\left(\left[R_{i}\right]\right)$ matrices are the ordinary pointgroup matrices for the various irreducible representations of the point group $D_{3}$. The charactertable for the $D_{3}$ point group is shown in Table III.

As all elements of the group belong to the group of the wave vector $\overrightarrow{\mathrm{k}}_{1}, s$ is equal to 1 , so the representation matrices only have one block [see Eq. (2.27)]. Therefore, we have

$$
\underline{D}\left(\left[S_{i} \mid \overrightarrow{\mathrm{t}}_{i}\right]\right)=1 \times \underline{\Gamma}\left(\left[S_{i}\right]\right) .
$$

TABLE II. Multiplication table of the rotational part of the symmetry elements of the $D_{3}^{6}$ space group.

\begin{tabular}{l|llllll}
\hline & $S_{1}$ & $S_{3}$ & $S_{5}$ & $S_{7}$ & $S_{9}$ & $S_{11}$ \\
\hline$S_{1}$ & $S_{1}$ & $S_{3}$ & $S_{5}$ & $S_{7}$ & $S_{9}$ & $S_{11}$ \\
$S_{3}$ & $S_{3}$ & $S_{5}$ & $S_{1}$ & $S_{9}$ & $S_{11}$ & $S_{7}$ \\
$S_{5}$ & $S_{5}$ & $S_{1}$ & $S_{3}$ & $S_{11}$ & $S_{7}$ & $S_{9}$ \\
$S_{7}$ & $S_{7}$ & $S_{11}$ & $S_{9}$ & $S_{1}$ & $S_{5}$ & $S_{3}$ \\
$S_{9}$ & $S_{9}$ & $S_{7}$ & $S_{11}$ & $S_{3}$ & $S_{1}$ & $S_{5}$ \\
$S_{11}$ & $S_{11}$ & $S_{9}$ & $S_{7}$ & $S_{5}$ & $S_{3}$ & $S_{1}$ \\
\hline \hline
\end{tabular}

From the charactertable for $D_{3}$ we see, that for the $\Gamma_{1}$ and the $\Gamma_{2}$ representations, the $\Gamma\left(\left[S_{i}\right]\right)$ matrices are one dimensional (that is $d=1$ ) and for the $\Gamma_{3}$ representation the $\Gamma\left(\left[S_{i}\right]\right)$ matrices are two dimensional (that is $d=2)$. The matrices are given in Table III for $D_{3}$.

$$
\text { 2. } \overrightarrow{\mathrm{k}}_{1}=\mu \cdot \overrightarrow{\mathrm{a}}_{3}\left(0<\mu<\pi /\left|\overrightarrow{\mathrm{a}}_{3}\right|\right)
$$

This is a point inside the Brillouin zone. From the definition of the symmetry operations it is easy to see that the group of the wave vector consists of the elements

$$
G_{\overrightarrow{\mathrm{k}}_{1}}:\left[S_{1} \mid \underline{0}\right],\left[S_{3} \mid \frac{1}{3} \overrightarrow{\mathrm{a}}_{3}\right],\left[S_{5} \mid \frac{2}{3} \overrightarrow{\mathrm{a}}_{3}\right],
$$

and therefore

$$
G=G_{\overrightarrow{\mathrm{k}}_{1}}+\left[S_{7} \mid \underline{0}\right] G_{\overrightarrow{\mathrm{k}}_{1}} .
$$

As the elements $\left[S_{7} \mid 0\right],\left[S_{9} \mid \frac{1}{3} \vec{a}_{3}\right]$, and $\left[S_{11} \mid \frac{2}{3} \vec{a}_{3}\right]$ all turn $\overrightarrow{\mathrm{k}}_{1} \rightarrow-\overrightarrow{\mathrm{k}}_{1}$, we only have to consider one of these elements in the coset development. It is also very easily verified, that the development in (3.1) includes all elements of $G$, as it should. (Remember that $T$ is a subgroup of $G_{\overrightarrow{\mathrm{k}}_{1}}$.) Thus, we see that $s=2$, so the representation matrices are $2 \times 2$ block matrices [see (2.27)]. Let us systematize our analysis. The first step is to rewrite Eq. (2.37) in the form

$$
\left[P_{m} \mid \overrightarrow{\mathrm{p}}_{m}\right]^{-1}\left[S_{i} \mid \overrightarrow{\mathrm{t}}_{i}\right]\left[P_{l} \mid \overrightarrow{\mathrm{p}}_{l}\right]=\left[R_{n} \mid \overrightarrow{\mathrm{r}}_{n}\right]
$$

We perform the multiplications on the left-hand side in two steps. Firstly, we write a multiplication table for

$$
\left[P_{m} \mid \overrightarrow{\mathrm{p}}_{m}\right]^{-1}\left[S_{i} \mid \overrightarrow{\mathrm{t}}_{i}\right], \quad i=1, \ldots, 6, \quad m=1,2 .
$$

Secondly, when we want to find the representation of the $i$ th symmetry operation $\left[S_{i} \mid \vec{t}_{i}\right]$, we take the

\begin{tabular}{|c|c|c|c|c|c|c|}
\hline$D_{3}$ & $S_{1}$ & $S_{3}$ & $S_{5}$ & $S_{7}$ & $S_{9}$ & $S_{11}$ \\
\hline$\Gamma_{1}$ & 1 & 1 & 1 & 1 & 1 & 1 \\
\hline$\Gamma_{2}$ & 1 & 1 & 1 & -1 & -1 & -1 \\
\hline$\Gamma$ & 10 & $\epsilon^{a} 0$ & $\epsilon^{* b} 0$ & 01 & $0 \epsilon$ & $0 \epsilon^{*}$ \\
\hline${ }^{1} 3$ & 01 & $0 \epsilon^{*}$ & $0 \epsilon$ & 10 & $\epsilon^{*} 0$ & $\epsilon 0$ \\
\hline
\end{tabular}
elements in the $i$ th column of the multiplication table just generated, and make a new multiplication table for the multiplication of the $i$ th column with the elements $\left[P_{l} \mid \overrightarrow{\mathrm{p}}_{l}\right]$. Then according to Eq. (3.2) we should find just one element from $G_{\vec{k}_{1}}$ in each

TABLE III. Character table for the $D_{3}$ point group [from Kovalev (Ref. 7)].

${ }^{a} \epsilon=\exp \left(\frac{2}{3} i \pi\right)$.

${ }^{b} \epsilon^{*}=\exp \left(-\frac{2}{3} i \pi\right)$. 
column and row of this multiplication table. The row $(m)$ and column $(l)$ in which this element is found corresponds to the block in the representation matrix, (2.27), which has to be different from zero, and the block is given according to Eq. (2.38). It may seem difficult, so let us see how it works. According to our notation we have

$$
\left[R_{1} \mid \overrightarrow{\mathrm{r}}_{1}\right]=\left[S_{1} \mid \overrightarrow{\mathbf{b}}\right], \quad\left[R_{2} \mid \overrightarrow{\mathrm{r}}_{2}\right]=\left[S_{3} \mid \frac{1}{3} \overrightarrow{\mathrm{a}}_{3}\right]
$$

\begin{tabular}{|c|c|c|c|c|c|c|c|}
\hline$\left[P_{m} \mid \overrightarrow{\mathrm{p}}_{m}\right]^{-1}$ & $\frac{i}{m}$ & {$\left[\begin{array}{c}{\left[S_{1} \mid \overrightarrow{0}\right]} \\
1\end{array}\right.$} & $\begin{array}{c}{\left[S_{3} \mid \frac{1}{3} \vec{a}_{3}\right]} \\
2\end{array}$ & $\begin{array}{c}{\left[S_{5} \mid \frac{2}{3} \vec{a}_{3}\right]} \\
3\end{array}$ & $\begin{array}{c}{\left[S_{7} \mid \overrightarrow{0}\right]} \\
4\end{array}$ & $\begin{array}{c}{\left[S_{9} \mid \frac{1}{3} \vec{a}_{3}\right]} \\
5 \\
\end{array}$ & $\begin{array}{c}{\left[S_{11} \mid \frac{2}{3} \overrightarrow{\mathrm{a}}_{3}\right]} \\
6 \\
\end{array}$ \\
\hline$\left[S_{1} \mid \overrightarrow{0}\right]$ & 1 & $\overrightarrow{\left[s_{1} \mid \overrightarrow{0}\right]}$ & {$\left[S_{3} \mid \frac{1}{3} \vec{a}_{3}\right]$} & {$\left[S_{5} \mid \frac{2}{3} \overrightarrow{\mathrm{a}}_{3}\right]$} & {$\left[S_{7} \mid 0\right]$} & {$\left[S_{9} \mid \frac{1}{3} \overrightarrow{\mathrm{a}}_{3}\right]$} & {$\left[S_{11} \mid \frac{2}{3} \overrightarrow{\mathrm{a}}_{3}\right]$} \\
\hline$\left[S_{7} \mid \overrightarrow{0}\right]$ & 2 & {$\left[s_{7} \mid \overrightarrow{0}\right]$} & {$\left[S_{11} \mid-\frac{1}{3} \vec{a}_{3}\right]$} & {$\left[S_{9} \mid-\frac{2}{3} \vec{a}_{3}\right]$} & {$\left[S_{1} \mid 0\right]$} & {$\left[S_{5} \mid-\frac{1}{3} \vec{a}_{3}\right]$} & {$\left[S_{3} \mid-\frac{2}{3} \vec{a}_{3}\right]$} \\
\hline
\end{tabular}

The representation of the symmetry elements of the $D_{3}^{6}$ group are then found to be $\left[S_{1} \mid \overrightarrow{0}\right]$ or $[\epsilon \mid \overrightarrow{0}]$ :

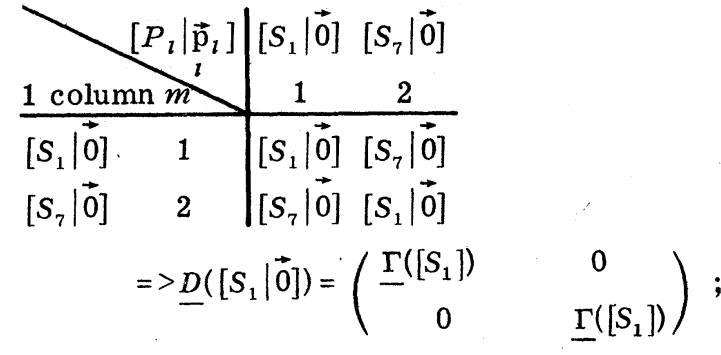

$\left[S_{3} \mid \frac{1}{3} \vec{a}_{3}\right]:$

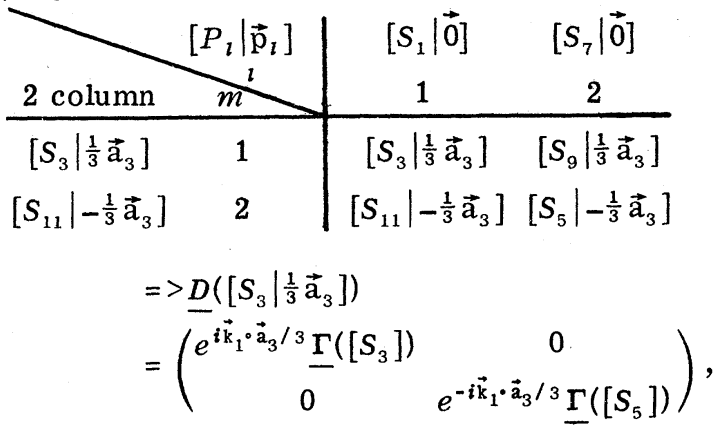

and so on. Let us just list the results for the other operations:

$$
\begin{aligned}
& {\left[S_{5} \mid \frac{2}{3} \overrightarrow{\mathrm{a}}_{3}\right]: .} \\
& \underline{D}\left(\left[S_{5} \mid \frac{2}{3} \overrightarrow{\mathrm{a}}_{3}\right]\right) \\
& \quad=\left(\begin{array}{cc}
e^{i \overrightarrow{\mathrm{k}}_{1} \cdot 2 \overrightarrow{\mathrm{a}}_{3} / 3} \underline{\Gamma\left(\left[S_{5}\right]\right)} & 0 \\
0 & e^{-i \overrightarrow{\mathrm{k}}_{1} \cdot 2 \overrightarrow{\mathrm{a}}_{3} / 3} \underline{\Gamma\left(\left[S_{3}\right]\right)}
\end{array}\right) ;
\end{aligned}
$$

$\left[S_{7} \mid \overrightarrow{0}\right]:$

$\underline{D}\left(\left[S_{7} \mid \overrightarrow{0}\right]\right)=\left(\begin{array}{cc}0 & \Gamma\left(\left[S_{1}\right]\right) \\ \underline{\Gamma}\left(\left[S_{1}\right]\right) & 0\end{array}\right)$

$$
\begin{aligned}
& {\left[R_{3} \mid \overrightarrow{\mathrm{r}}_{3}\right]=\left[S_{5} \mid \frac{2}{3} \overrightarrow{\mathrm{a}}_{3}\right]} \\
& \left.\left[P_{1} \mid \overrightarrow{\mathrm{p}}_{1}\right]=S_{1} \mid \overrightarrow{\mathrm{O}}\right], \quad\left[P_{2} \mid \overrightarrow{\mathrm{p}}_{2}\right]=\left[S_{7} \mid \overrightarrow{\mathrm{O}}\right], \\
& {\left[P_{1} \mid \overrightarrow{\mathrm{p}}_{1}\right]^{-1}=\left[S_{1} \mid \overrightarrow{\mathrm{O}}\right] ;\left[P_{2} \mid \overrightarrow{\mathrm{p}}_{2}\right]^{-1}=\left[S_{7} \mid \overrightarrow{\mathrm{O}}\right] .}
\end{aligned}
$$

We have used

$$
\left[P_{i} \mid \overrightarrow{\mathrm{p}}_{i}\right]^{-1}=\left[P_{i}^{-1} \mid-P_{i} \overrightarrow{\mathrm{p}}_{i}\right] \text {. }
$$

From these definitions we find, using Table II,

$$
\begin{aligned}
& {\left[S_{9} \mid \frac{1}{3} \overrightarrow{\mathrm{a}}_{3}\right]:} \\
& \underline{D}\left(\left[S_{9} \mid \frac{1}{3} \overrightarrow{\mathrm{a}}_{3}\right]\right)=\left(\begin{array}{cc}
0 & e^{i \overrightarrow{\mathrm{k}}_{1} \cdot \overrightarrow{\mathrm{a}}_{3} / 3} \underline{\Gamma\left(\left[S_{5}\right]\right)} \\
e^{-i \overrightarrow{\mathrm{k}}_{1} \cdot \overrightarrow{\mathrm{a}}_{3} / 3} \underline{\Gamma}\left(\left[S_{3}\right]\right) & 0
\end{array}\right) ; \\
& {\left[S_{11} \mid \frac{2}{3} \overrightarrow{\mathrm{a}}_{3}\right]:} \\
& \underline{D}\left(\left[S_{11} \mid \frac{2}{3} \vec{a}_{3}\right]\right) \\
& =\left(\begin{array}{cc}
0 & e^{i \overrightarrow{\mathrm{k}}_{1} \cdot 2 \overrightarrow{\mathrm{a}}_{3} / 3} \underline{\Gamma\left(\left[S_{5}\right]\right)} \\
e^{-i \overrightarrow{\mathrm{k}}_{1} \circ 2 \overrightarrow{\mathrm{a}}_{3} / 3} \underline{\Gamma\left(\left[S_{3}\right]\right)} & 0
\end{array}\right) . \\
& \text { 3. } \overrightarrow{\mathrm{k}}_{1}=\mu \cdot \overrightarrow{\mathrm{a}}_{3}, \mu=\pi /\left|\overrightarrow{\mathrm{a}}_{3}\right|
\end{aligned}
$$

This is a point at the surface of the Brillouin zone. From the definition of the symmetry operations it is easy to see that no operation changes $\vec{k}_{1}$ in an essential way, that is $G_{\overrightarrow{\mathrm{k}}_{1}}=G$. Therefore $s$ $=1$, so the representation matrices are $1 \times 1$ block matrices (2.27), and they are given according to

$$
\underline{D}\left(\left[S_{i} \mid \overrightarrow{\mathrm{t}}_{i}\right]\right)=e^{i \overrightarrow{\mathrm{k}}_{1} \cdot \overrightarrow{\mathrm{t}}_{i}} \underline{\Gamma}\left(\left[S_{i}\right]\right) .
$$

As we are at the surface of the Brillouin zone, the $\Gamma\left(\left[S_{i}\right]\right)$ matrices are no longer the point-group representation matrices. This is easily seen by a consideration of the multiplication rules for the $\Gamma\left(\left[S_{i}\right]\right)$ matrices. Consider, for example,

$$
\left[S_{7} \mid \overrightarrow{0}\right]\left[S_{11} \mid \frac{2}{3} \vec{a}_{3}\right]=\left[S_{1} \mid-\vec{a}_{3}\right]\left[S_{3} \mid \frac{1}{3} \vec{a}_{3}\right] \text {. }
$$

Inserting the representation matrices (3.3), we find

$$
\underline{\Gamma}\left(\left[S_{7}\right]\right) e^{i \overrightarrow{\mathrm{k}}_{1} \cdot 2 \overrightarrow{\mathrm{a}}_{3} / 3} \underline{\Gamma}\left(\left[S_{11}\right]\right)=e^{-i \overrightarrow{\mathrm{k}}_{1} \cdot 2 \overrightarrow{\mathrm{a}}_{3} / 3} \underline{\Gamma}\left(\left[S_{3}\right]\right) .
$$

From this we deduce the multiplication rule

$$
\begin{aligned}
\underline{\Gamma}\left(\left[S_{7}\right]\right) \underline{\Gamma}\left(\left[S_{11}\right]\right) & =\exp \left\{i \overrightarrow{\mathrm{k}}_{1} \cdot\left[S_{7}\left(\frac{2}{3} \overrightarrow{\mathrm{a}}_{3}-\frac{2}{3} \overrightarrow{\mathrm{a}}_{3}\right)\right]\right\} \underline{\Gamma\left(\left[S_{3}\right]\right)} \\
& =\exp \left[i \overrightarrow{\mathrm{k}}_{1} \cdot(-) \frac{4}{3} \overrightarrow{\mathrm{a}}_{3}\right] \underline{\Gamma}\left(\left[S_{3}\right]\right),
\end{aligned}
$$

which shows that the $\Gamma\left(\left[S_{i}\right]\right)$ matrices are the multiplier representations of the point group $D_{3}$ [compare with Eq. (2.39)]. 
4. $\mathrm{k}_{1}$ in a general position (no symmetry direction)

Then we have $G_{\overrightarrow{\mathrm{k}}_{1}}=T$, the translation group, and we have

$$
\begin{aligned}
& {\left[R_{1} \mid \overrightarrow{\mathrm{r}}_{1}\right]=\left[S_{1} \mid \overrightarrow{0}\right],} \\
& {\left[P_{1} \mid \overrightarrow{\mathrm{p}}_{1}\right]=\left[S_{1} \mid \overrightarrow{0}\right],\left[P_{1} \mid \overrightarrow{\mathrm{p}}_{1}\right]^{-1}=\left[S_{1} \mid \overrightarrow{0}\right],\left[P_{2} \mid \overrightarrow{\mathrm{p}}_{2}\right]=\left[S_{3} \mid \frac{1}{3} \overrightarrow{\mathrm{a}}_{3}\right], \quad\left[P_{2} \mid \overrightarrow{\mathrm{p}}_{2}\right]^{-1}=\left[S_{5} \mid-\frac{1}{3} \overrightarrow{\mathrm{a}}_{3}\right],} \\
& {\left[P_{3} \mid \overrightarrow{\mathrm{p}}_{3}\right]=\left[S_{5} \mid \frac{2}{3} \overrightarrow{\mathrm{a}}_{3}\right],\left[P_{3} \mid \overrightarrow{\mathrm{p}}_{3}\right]^{-1}=\left[S_{3} \mid-\frac{2}{3} \overrightarrow{\mathrm{a}}_{3}\right], \quad\left[P_{4} \mid \overrightarrow{\mathrm{p}}_{4}\right]=\left[S_{7} \mid \overrightarrow{0}\right],\left[P_{4} \mid \overrightarrow{\mathrm{p}}_{4}\right]^{-1}=\left[S_{7} \mid \overrightarrow{0}\right],} \\
& {\left[P_{5} \mid \overrightarrow{\mathrm{p}}_{5}\right]=\left[S_{9} \mid \frac{1}{3} \overrightarrow{\mathrm{a}}_{3}\right],\left[P_{5} \mid \overrightarrow{\mathrm{p}}_{5}\right]^{-1}=\left[S_{9} \mid \frac{1}{3} \overrightarrow{\mathrm{a}}_{3}\right],\left[P_{6} \mid \overrightarrow{\mathrm{p}}_{6}\right]=\left[S_{11} \mid \frac{2}{3} \overrightarrow{\mathrm{a}}_{3}\right], \quad\left[P_{6} \mid \overrightarrow{\mathrm{p}}_{6}\right]^{-1}=\left[S_{11} \mid \frac{2}{3} \overrightarrow{\mathrm{a}}_{3}\right],}
\end{aligned}
$$

that is, our representation matrices are $6 \times 6$ block matrices. The procedure for finding the representations are exactly the same as already described.

We will not repeat that here, but just give the representation for one operation, say $\left[S_{5} \mid \frac{2}{3} \vec{a}_{3}\right]$. We find

$$
\underline{D}\left(\left[S_{5} \mid \frac{2}{3} \vec{a}_{3}\right]\right)=\left[\begin{array}{cccccc}
0 & f_{1} \underline{\Gamma}\left(\left[S_{1}\right]\right) & 0 & 0 & 0 & 0 \\
0 & 0 & f_{1} \underline{\Gamma\left(\left[S_{1}\right]\right)} & 0 & 0 & 0 \\
\Gamma\left(\left[S_{1}\right]\right) & 0 & 0 & 0 & 0 & 0 \\
0 & 0 & 0 & 0 & f_{1}^{*} \underline{\Gamma\left(\left[S_{1}\right]\right)} & 0 \\
0 & 0 & 0 & 0 & 0 & f_{1}^{*} \underline{\Gamma\left(\left[S_{1}\right]\right)} \\
0 & 0 & 0 & \underline{\Gamma}\left(\left[S_{1}\right]\right) & 0 & 0
\end{array}\right] \text {, }
$$

where $f_{1}=\exp \left(i \overrightarrow{\mathrm{k}}_{1} \cdot \overrightarrow{\mathrm{a}}_{3}\right), f_{1}^{*}=\exp \left(-i \overrightarrow{\mathrm{k}}_{1} \cdot \overrightarrow{\mathrm{a}}_{3}\right)$, and $\Gamma\left(\left[S_{1}\right]\right)=1$.

Before we close this subsection, let us for a moment stop and ask what we have accomplished, and what all these matrices really mean.

We have seen that the dimension (the number of blocks) of the representation matrices depends on the vector $\overrightarrow{\mathrm{k}}_{1}$. The $\Gamma\left(\left[S_{i}\right]\right)$ matrices ought to be indexed as $\Gamma\left(\left[S_{i}\right]\right)_{j}$, where the subscript $j$ denotes which irreducible representation we consider. For example, in the case $\mu=0$, we saw that the point

$$
\begin{aligned}
& \psi_{11}, \ldots, \psi_{1 d} \text { : transforms according to } \overrightarrow{\mathrm{k}}_{1} \text { under } T \text {, } \\
& \psi_{21}, \ldots, \psi_{2 d}: \text { transforms according to } P_{2} \overrightarrow{\mathrm{k}}_{1} \text { under } T \text {, } \\
& \text {. } . \text {. } \text {. }
\end{aligned}
$$

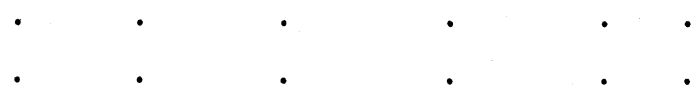

$$
\begin{aligned}
& \psi_{s 1}, \ldots, \psi_{s d}: \text { transforms according to } P_{s} \overrightarrow{\mathrm{k}}_{1} \text { under } T \text {, }
\end{aligned}
$$

where

$$
\psi_{\nu \mu}=\left[P_{\nu} \mid \overrightarrow{\mathrm{p}}_{\nu}\right] \psi_{1 \mu}, \quad \nu=1, s \text { and } \mu=1, d
$$

$d$ is the dimension of a block. As with the $\Gamma\left(\left[S_{i}\right]\right)$ matrices, this basis set also ought to be indexed to tell which irreducible representations they span. The basis sets will of course differ when they span different representations. We have not used the in- group $D_{3}$ had two one-dimensional and one two-dimensional irreducible representations. In the expression for the representation matrices we should therefore have indexed the $\Gamma\left(\left[S_{i}\right]\right)$ matrix as $\Gamma\left(\left[S_{i}\right]\right)_{\Gamma_{1}}, \Gamma\left(\left[S_{i}\right]\right)_{\Gamma_{2}}, \Gamma\left(\left[S_{i}\right]\right)_{\Gamma_{3}}$, respectively. We have omitted this index, because it is rather obvious that it has to be so, and also in order not to drown in indices.

It should be remembered that, if there are $s$ blocks, then the matrices above are represented in a basis set like 
$\left[S_{9} \mid \frac{1}{3} \overrightarrow{\mathrm{a}}_{3}\right] \psi=\left[\psi_{11}, \ldots, \psi_{1 d}, \psi_{21}, \ldots, \psi_{2 d}, \psi_{2 d+1}, \ldots, \psi_{n}\right]\left(\begin{array}{cc}\overrightarrow{\mathrm{k}}_{1} \cdot \overrightarrow{\mathrm{a}}_{3} / 3 \\ \underline{\Gamma\left(\left[R_{3}\right]\right)} \\ e^{-i \overrightarrow{\mathrm{k}}_{1} \cdot \overrightarrow{\mathrm{a}}_{3} / 3} \underline{\underline{\Gamma}\left(\left[R_{5}\right]\right)} & 0\end{array}\right)\left(\begin{array}{c}s_{1} \\ \cdot \\ \cdot \\ s_{d} \\ 0 \\ \cdot \\ \cdot \\ \cdot \\ 0\end{array}\right]$

B. Generation of the multiplier representation of point group $D_{3}$

We have seen that the $\Gamma\left(\left[R_{i}\right]\right)$ matrices for $\overrightarrow{\mathrm{k}}_{1}$ $=\pi /\left|\vec{a}_{3}\right|$, which is on the Brillouin-zone surface, are multiplier representations of $D_{3}$. For each operation in $D_{3}$ we therefore have to find an appropriate phase factor, which has to be multiplied with all the representation matrices of that operation known from the ordinary representation of $D_{3}$.

The phase factors are found using the multiplication table for the rotational elements of $D_{3}$ and a multiplication table generated from (2.39). We will work with the full symmetrical one-dimensional representation, since we have

$$
\underline{\Gamma}\left(\left[R_{j}\right]\right)=1
$$

for all $j$. Then $\underline{\Gamma}\left(\left[R_{j}\right]\right)$ may generally be written

$$
\Gamma\left(\left[R_{j}\right]\right)=e^{i \theta_{j}}
$$

In order to have a shorthand notation for the $\Gamma\left(\left[R_{j}\right]\right)$ matrix, we write

$$
\underline{\Gamma}\left(\left[R_{j}\right]\right) \sim \underline{\Gamma}_{j} .
$$

We have already generated the multiplication table for the rotational elements of $D_{3}$; now let us generate the other multiplication table needed, using our shorthand notation (3.6). The result is shown in Table IV.

From Table IV we see

$$
\begin{aligned}
& \Gamma_{7} \Gamma_{7}=\Gamma_{1}=1 \Rightarrow \Gamma_{7}=1, \\
& \Gamma_{3} \Gamma_{5}=\Gamma_{1}=1 \Rightarrow \Gamma_{3}=\Gamma_{5}^{*}, \Gamma_{5}=\Gamma_{3}^{*}, \\
& \Gamma_{3} \Gamma_{3}=\Gamma_{5}=\Gamma_{3}^{*}
\end{aligned}
$$

or using (3.5)

$$
\begin{aligned}
& e^{i 2 \theta_{3}}=e^{-i \theta_{3}} \Rightarrow \theta_{3}=\frac{2}{3} \pi n, \quad \Gamma_{3}=e^{i 2 \pi \eta ! 3}, \\
& \Gamma_{3} \Gamma_{7}=\Gamma_{3} 1=\Gamma_{9} \Rightarrow \Gamma_{9}=\Gamma_{3}, \\
& \Gamma_{3} \Gamma_{9}=\Gamma_{3}^{2}=\Gamma_{11} \Rightarrow \Gamma_{11}=\Gamma_{3}^{2} .
\end{aligned}
$$

To decide which value of $n$ we are going to use, consider the following:

$$
\begin{aligned}
\Gamma_{7} \Gamma_{3} & =1 \times e^{i 2 \pi n / 3}=e^{-i 2 \pi / 3} \Gamma_{11} \\
& =e^{-i 2 \pi / 3} e^{i 4 \pi n / 3} .
\end{aligned}
$$

From this we may determine $n$,

$$
\frac{2}{3} \pi n=\frac{4}{3} \pi n-\frac{2}{3} \pi
$$

or $n=1$. The phase factors which have to be multiplied against the ordinary representation matrices of $D_{3}$ are, according to the analysis given above,

$$
\begin{array}{lll}
\Gamma_{1}=1, & \Gamma_{3}=e^{i 2 \pi / 3}, & \Gamma_{5}=e^{-i 2 \pi / 3}, \\
\Gamma_{7}=1, & \Gamma_{9}=e^{i 2 \pi / 3}, & \Gamma_{11}=e^{-i 2 \pi / 3} .
\end{array}
$$

This gives the multiplier representation of $D_{3}$ as shown in Table $\mathrm{V}$. This is just the same table as found in Kovalev. ${ }^{7}$ Yet, the results are not quite identical, because everywhere that we have $\epsilon$, he has $\epsilon^{*}$ and vice versa. This has no physical implications and as already pointed out, the differences are due to the definitions (2.40) and (2.41). If we had used the same sign as Kovalev, then Eq. (3.7) would have been

$$
\frac{2}{3} \pi n=\frac{4}{3} \pi n+\frac{2}{3} \pi,
$$

which gives $n=-1$ and we would have obtained Kovalev's results.

TABLE IV. Multiplication table for the $D_{3}^{6}$ space group based on Eq. (2.39). $\Gamma_{i}$ is a shorthand notation for the matrices $\Gamma\left(\left[R_{i}\right]\right) . \overrightarrow{\mathrm{k}}_{1}=\left(\pi /\left|\overrightarrow{\mathrm{a}}_{3}\right|\right) \overrightarrow{\mathrm{a}}_{3}$.

\begin{tabular}{l|llllll}
\hline \hline & $\Gamma_{1}$ & $\Gamma_{3}$ & $\Gamma_{5}$ & $\Gamma_{7}$ & $\Gamma_{9}$ & $\Gamma_{11}$ \\
\hline$\Gamma_{1}$ & $\Gamma_{1}$ & $\Gamma_{3}$ & $\Gamma_{5}$ & $\Gamma_{7}$ & $\Gamma_{9}$ & $\Gamma_{11}$ \\
$\Gamma_{3}$ & $\Gamma_{3}$ & $\Gamma_{5}$ & $\Gamma_{1}$ & $\Gamma_{9}$ & $\Gamma_{11}$ & $\Gamma_{7}$ \\
$\Gamma_{5}$ & $\Gamma_{5}$ & $\Gamma_{1}$ & $\Gamma_{3}$ & $\Gamma_{11}$ & $\Gamma_{7}$ & $\Gamma_{9}$ \\
$\Gamma_{7}$ & $\Gamma_{7}$ & $\Gamma_{11} f_{1}{ }^{a}$ & $\Gamma_{9} f_{2}{ }^{b}$ & $\Gamma_{1}$ & $\Gamma_{5} f_{1}$ & $\Gamma_{3} f_{2}$ \\
$\Gamma_{9}$ & $\Gamma_{9}$ & $\Gamma_{7} f_{1}$ & $\Gamma_{11} f_{2}$ & $\Gamma_{3}$ & $\Gamma_{1} f_{2}$ & $\Gamma_{5} f_{2}$ \\
$\Gamma_{11}$ & $\Gamma_{11}$ & $\Gamma_{9} f_{1}$ & $\Gamma_{7} f_{2}$ & $\Gamma_{5}$ & $\Gamma_{3} f_{1}$ & $\Gamma_{1} f_{2}$ \\
\hline
\end{tabular}

${ }^{a} f_{1}=\exp \left(-i \overrightarrow{\mathrm{k}}_{1} \cdot \frac{2}{3} \overrightarrow{\mathrm{a}}_{3}\right)$.

${ }^{\mathrm{b}} f_{2}=\exp \left(-i \overrightarrow{\mathrm{k}}_{1} \cdot \frac{4}{3} \vec{a}_{3}\right)$. 
TABLE V. Multiplier representation of the $D_{3}$ point group.

\begin{tabular}{|c|c|c|c|c|c|c|}
\hline$D_{3}$ & $S_{1}$ & $S_{3}$ & $S_{5}$ & $S_{7}$ & $S_{9}$ & $S_{11}$ \\
\hline$\Gamma_{1}$ & 1 & $\epsilon^{\mathbf{a}}$ & $\epsilon^{* b}$ & 1 & $\epsilon$ & $\epsilon^{*}$ \\
\hline$\Gamma_{2}$ & 1 & $\epsilon$ & $\epsilon^{*}$ & -1 & $-\epsilon$ & $-\epsilon^{*}$ \\
\hline$\Gamma_{3}$ & 10 & $\epsilon^{*} 0$ & $\epsilon 0$ & 01 & $0 \epsilon^{*}$ & $0 \epsilon$ \\
\hline 3 & 01 & $\begin{array}{ll}0 & 1\end{array}$ & 0.1 & 10 & 10 & 10 \\
\hline
\end{tabular}

\section{Generation of symmetry coordination for trigonal selenium}

The symmetry coordinates are displacement patterns of the atoms in the unit cell of a certain symmetry. They play an important role in lattice-dynamical calculations. Thus the dynamical matrix will be on a simple block form and therefore very often easier to analyze, if symmetry coordinates are used to express the potential energy instead of simple Cartesian displacements of the atoms; one may assign the proper symmetry to the various frequencies, a feature of great importance in cases where branches are crossing.

The symmetry coordinates are found by letting the projection operator (2.42) work on arbitrary displacement patterns of the atoms in the unit cell. The result of this operation will be either no displacements of any atom or a displacement pattern of the right symmetry.

Let us digress for a moment and briefly discuss the structure of trigonal selenium. The structure is shown in Fig. 1, where also are shown the lattice bas is vectors $\left(\vec{a}_{1}, \vec{a}_{2}, \vec{a}_{3}\right)$, a Cartesian-coordinate system $(\vec{i}, \vec{j}, \vec{k})$ used to define absolute atomic positions, and the lattice vectors of the reciprocal lattice $\left(\vec{b}_{1}, \vec{b}_{2}, \vec{b}_{3}\right)$. The atomic positions in the unit cell are shown in Table VI. The relation between the lattice basis vectors $\left(\vec{a}_{1}, \vec{a}_{2}, \vec{a}_{3}\right)$ and the Cartesian basis vector $(\vec{i}, \vec{j}, \vec{k})$ is

$$
\begin{array}{r}
\left(\overrightarrow{\mathrm{a}}_{1}, \overrightarrow{\mathrm{a}}_{2}, \overrightarrow{\mathrm{a}}_{3}\right)=(\overrightarrow{\mathrm{i}}, \overrightarrow{\mathrm{j}}, \overrightarrow{\mathrm{k}})\left[\begin{array}{ccc}
c_{1} & -\frac{1}{2} c_{1} & 0 \\
0 & \frac{1}{2} \sqrt{3} c_{1} & 0 \\
0 & 0 & c_{3}
\end{array}\right], \\
c_{1}=4.3712, c_{3}=4.953899 .
\end{array}
$$

It is seen that trigonal selenium consists of long spiral chains arranged in a hexagonal pattern, so the Bravais lattice becomes hexagonal. In Table I we have already listed the symmetry operations. The small atomic displacements from the equilibrium positions are represented in local Cartesiancoordinate systems oriented in the same way as the $(\vec{i}, \vec{j}, \vec{k})$ system, and with origins at the various equilibrium positions of the atoms. To describe
TABLE VI. Atomic positions in the unit cell of trigonal selenium represented in the lattice basis vector system, $\vec{a}_{1}, \vec{a}_{2}, \vec{a}_{3}$.

\begin{tabular}{cc}
\hline Atom No. & $\begin{array}{c}\text { Position [represented in } \\
\text { the }\left(\vec{a}_{1}, \vec{a}_{2}, \vec{a}_{3}\right) \text { system] }\end{array}$ \\
\hline 1 & $\left(x, 0,-\frac{1}{3}\right)^{\mathrm{a}}$ \\
2 & $(0, x, 0)$ \\
3 & $\left(-x,-x, \frac{1}{3}\right)$ \\
\hline
\end{tabular}

${ }^{\mathrm{a}} x=0.2254$.

the displacements of the three atoms in the unit cell, we thus need nine coordinate axis, namely,

$$
\left(\vec{i}_{1} \vec{j}_{1} \vec{k}_{1}, \vec{i}_{2} \vec{j}_{2} \vec{k}_{2}, \vec{i}_{3} \vec{j}_{3} \vec{k}_{3}\right) \text {. }
$$

We now have to represent the matrices $M$ and $L$ [Eq. (2.43)] in this basis-coordinate system. The dimension of the matrices becomes $9 \times 9$, and they are constructed in such a way that they give the result of the operation of $[S \mid \vec{t}]$ on an arbitrary displacement pattern $V$ according to

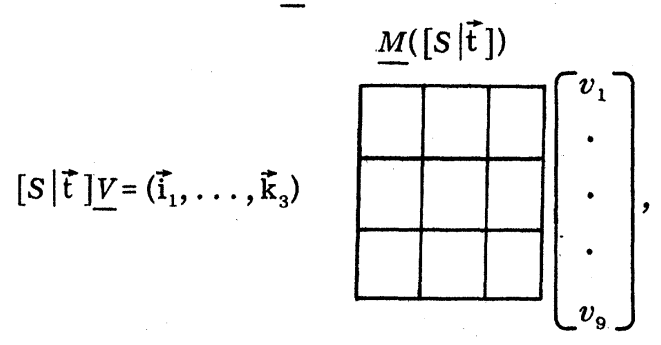

where $v_{i}$ are the components of $V$. We are now in the position to write a reducible representation of all symmetry operations of the group in the basis set (3.9). From (2.43) we see that the $M$ matrix is given by

$$
M([S \mid \overrightarrow{\mathrm{t}}])=\exp (i \overrightarrow{\mathrm{k}} \cdot \overrightarrow{\mathrm{t}}) \underline{L}([S]),
$$

where $L$ is a matrix just depending on the rotational part of the symmetry operation, and $\vec{k}$ is a wave vector. $L$ is a $3 \times 3$ block matrix [Eq. (3.10)], where each block is a $3 \times 3$ matrix, which either is the zero matrix or the representation matrix of the rotational part of the operation in the basis set of (3.9). These are very easy to obtain (see below).

If atom 1 is brought into the position of atom 2 by a symmetry operation, then the displacement of atom 1 is rotated as prescribed by the operation and transferred to the position of atom 2, the so-called active point of view. ${ }^{14}$ That means, according to Eq. (3.10), that the $(2,1)$ block of the representation matrix is different from the zero matrix and equal to the rotation matrix for the operation, while all other blocks in the first-column and the second-row blocks are zero matrices. It may also happen that a symmetry operation brings the displacement of an atom into the position of an atom 
outside the unit cell. From Fig. 1 we see that, for instance, the $\left[S_{3} \mid \frac{1}{3} \vec{a}_{3}\right]$ operation takes the displacement of atom 3 into the position of atom 1 in the cell above the unit cell going along the chain. If we call the displacement of atom 1 in the cell with position vector $\vec{R}_{h k l}$ for $V_{h k l}$, then we may represent $\underline{\mathrm{V}}_{h k l}$ in terms of basis set (3.9), $\mathrm{V}_{000}$, making use of the representation of a primitive translation from the appropriate cell to the unit cell. We find

$$
\underline{\mathrm{V}}_{h k l}=\underline{\mathrm{V}}_{000} \exp \left(i \overrightarrow{\mathrm{k}} \cdot \overrightarrow{\mathrm{R}}_{h k l}\right) \text {. }
$$

$\left[s_{1} \mid \overrightarrow{0}\right]:$

$$
\left[\begin{array}{llllllll}
1 & 0 & 0 & & & & & \\
0 & 1 & 0 & & 0 & & & 0 \\
0 & 0 & 1 & & & & & \\
& & & 1 & 0 & 0 & & \\
0 & & 0 & 1 & 0 & & 0 & \\
& & 0 & 0 & 1 & & & \\
& & & & & 1 & 0 & 0 \\
0 & & & 0 & & 0 & 1 & 0 \\
& & & & & 0 & 0 & 1
\end{array}\right], \operatorname{Tr}=9
$$

$\left[S_{3} \mid \frac{1}{3} \vec{a}_{3}\right]$ :

$$
\left[\begin{array}{c}
{\left[\begin{array}{ccc}
-\frac{1}{2} & -\frac{1}{2} \sqrt{3} & 0 \\
\frac{1}{2} \sqrt{3} & -\frac{1}{2} & 0 \\
0 & 0 & 1
\end{array}\right] f_{2}} \\
0 \\
0
\end{array}\left[\begin{array}{cccc}
-\frac{1}{2} & -\frac{1}{2} \sqrt{3} & 0 \\
\frac{1}{2} \sqrt{3} & -\frac{1}{2} & 0 \\
0 & 0 & 1
\end{array}\right] f_{2}\right.
$$

$\left[S_{5} \mid \frac{2}{3} \vec{a}_{3}\right]$ :

$$
\left[\begin{array}{c}
0 \\
0 \\
{\left[\begin{array}{cccc}
-\frac{1}{2} & \frac{1}{2} \sqrt{3} & 0 \\
-\frac{1}{2} \sqrt{3} & -\frac{1}{2} & 0 \\
0 & 0 & 1
\end{array}\right] f_{1}} \\
0
\end{array}\right.
$$

Therefore, if a symmetry operation brings the dislacement of an atom inside the unit cell into the position of an atom outside the unit cell, we just have to multiply the particular rotation matrix with the proper phase factor, before we fill out the relevant block in $L$. When all atoms have been investigated, we finally multiply the $L$ matrix with the phase factor in (3.11) and obtain $M$.

It is now a simple matter to write the representation matrices for the six symmetry operations in the basis set (3.9); they are 
$\left[S_{7} \mid \overrightarrow{0}\right]:$

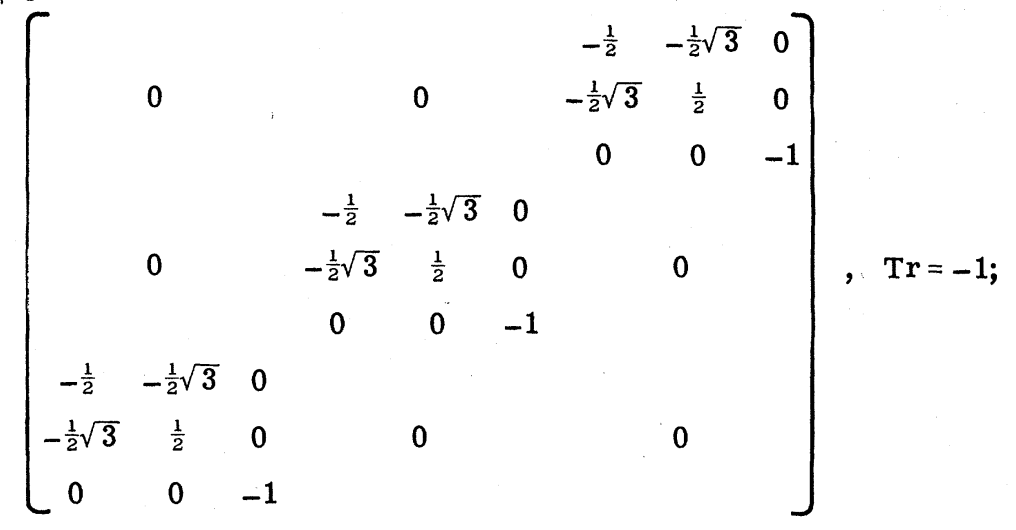

$\left[S_{9} \mid \frac{1}{3} \vec{a}_{3}\right]:$

$$
\begin{aligned}
& {\left[\begin{array}{ccc}
{\left[\begin{array}{ccc}
1 & 0 & 0 \\
0 & -1 & 0 \\
0 & 0 & -1
\end{array}\right] f_{1}^{*}} & 0 & 0 \\
0 & 0 & {\left[\begin{array}{ccc}
1 & 0 & 0 \\
0 & -1 & 0 \\
0 & 0 & -1
\end{array}\right] f_{2}}
\end{array}\right], \operatorname{Tr}=-f_{1}^{*} ;} \\
& {\left[\begin{array}{ccc}
1 & 0 & 0 \\
0 & -1 & 0 \\
0 & 0 & -1
\end{array}\right] f_{2} \quad 0 \quad}
\end{aligned}
$$

$\left[S_{11} \mid \frac{2}{3} \vec{a}_{3}\right]:$

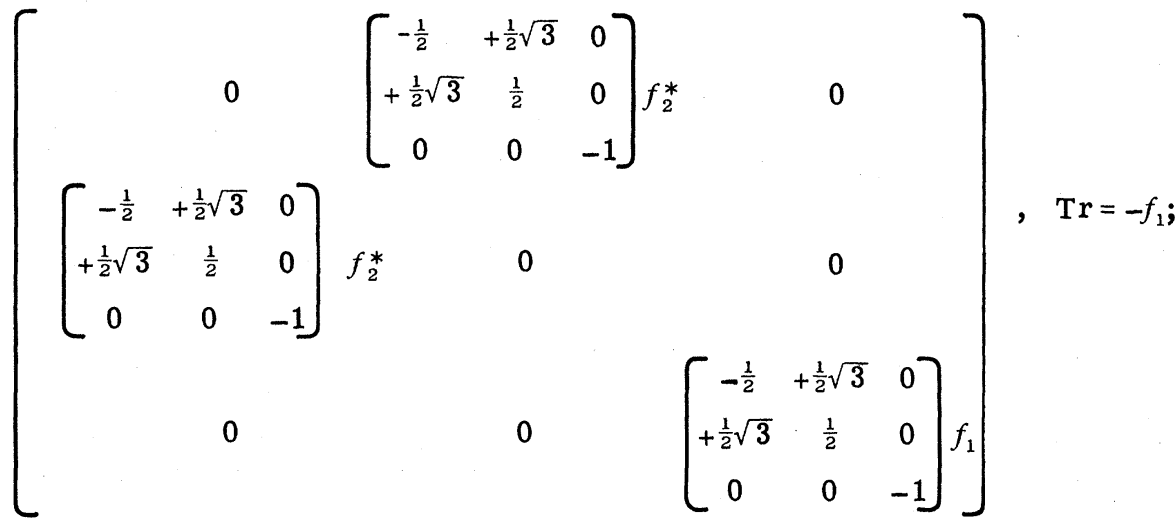

$$
\begin{aligned}
& f_{1}=\exp \left(2 i \vec{k} \cdot \frac{1}{3} \vec{a}_{3}\right), \\
& f_{2}=\exp \left(i \overrightarrow{\mathrm{k}} \cdot \frac{1}{3} \overrightarrow{\mathrm{a}}_{3}\right) \text {. }
\end{aligned}
$$

A superscript $*$ indicates the complex conjugate.

Before applying the projection-operator approach the wave vector $\overrightarrow{\mathrm{k}}$ to be considered must be chosen and its group determined. Wave-vector groups are found in Kovalev's book ${ }^{7}$ for a number of symmetry directions for all groups. In Table VII we have listed the various directions considered here, together with the character tables found in Kovalev's book. Note that the wave vectors $\overrightarrow{\mathrm{k}}$ are represented in the reciprocal-lattice basis system $\left(\vec{b}_{1}, \vec{b}_{2}, \vec{b}_{3}\right)$ (see Fig. 1).

To each character table we have added a column giving the number of symmetry coordinates, which span the various irreducible representations. This number is easily deduced from a well-known relation in ordinary point-group theory ${ }^{9,10}$ : 
TABLE VII. Character table for the group of the wave vector $\vec{k}$, for various directions in reciprocal space represented in the $\vec{b}_{1}, \vec{b}_{2}, \vec{b}_{3}$ coordinate system (from Kovalev).

\begin{tabular}{l|cccccc|c}
$K:$ & $\overrightarrow{\mathrm{k}}=\left(\frac{1}{3}, \frac{1}{3}, 0\right)$ \\
$\Gamma: \overrightarrow{\mathrm{k}}=(0,0,0)$
\end{tabular}

$\Delta: \overrightarrow{\mathrm{k}}=(0,0, \xi) ; 0<\xi<0.5$

$P: \overrightarrow{\mathrm{k}}=\left(\frac{1}{3}, \frac{1}{3}, \xi\right) ; 0<\xi<0.5$

\begin{tabular}{l|ccc|c}
\hline$\Delta, P$ & $h_{1}$ & $h_{3}$ & $h_{5}$ & Spanned by $s_{i}$ patterns \\
\hline$\Delta_{1}, P_{1}$ & 1 & 1 & 1 & 3 \\
$\Delta_{2}, P_{2}$ & 1 & $\epsilon$ & $\epsilon^{*}$ & 3 \\
$\Delta_{3}, P_{3}$ & 1 & $\epsilon^{*}$ & $\epsilon$ & 3 \\
\hline
\end{tabular}

$A: \overrightarrow{\mathrm{k}}=(0,0,0.5)$

$H: \overrightarrow{\mathrm{k}}=\left(\frac{1}{3}, \frac{1}{3}, 0.5\right)$

\begin{tabular}{|c|c|c|c|c|c|c|c|}
\hline$A, H$ & $h_{1}$ & $h_{5}$ & $h_{3}$ & $h_{7}$ & $h_{11}$ & $h_{9}$ & Spanned by $s_{i}$ patterns \\
\hline$\overline{A_{1}, H_{1}}$ & 1 & $\epsilon$ & $\epsilon^{*}$ & 1 & $\epsilon$ & $\epsilon^{*}$ & 1 \\
\hline$A_{2}, H_{2}$ & 1 & $\epsilon$ & $\epsilon^{*}$ & -1 & $-\epsilon$ & $-\epsilon^{*}$ & 2 \\
\hline$A_{3}, H_{3}$ & $\begin{array}{ll}1 & 0 \\
0 & 1\end{array}$ & $\begin{array}{ll}\epsilon^{*} & 0 \\
0 & 1\end{array}$ & $\begin{array}{ll}\epsilon & 0 \\
0 & 1\end{array}$ & $\begin{array}{ll}0 & 1 \\
1 & 0\end{array}$ & $\begin{array}{ll}0 & \epsilon^{*} \\
1 & 0\end{array}$ & $\begin{array}{l}0 \epsilon \\
10\end{array}$ & $3 \times 2=6$ \\
\hline
\end{tabular}

$T: \overrightarrow{\mathrm{k}}=(\xi, \xi, 0) ; 0<\xi<\frac{1}{3}$

\begin{tabular}{l|cc|c}
\hline$T$ & $h_{1}$ & $h_{11}$ & Spanned by $s_{i}$ patterns \\
\hline$T_{1}$ & 1 & 1 & 4 \\
$T_{2}$ & 1 & -1 & 5 \\
\hline
\end{tabular}

$S: \overrightarrow{\mathrm{k}}=(\xi, \xi, 0.5) ; 0<\xi<\frac{1}{3}$

\begin{tabular}{l|cc|c}
\hline$S$ & $h_{1}$ & $h_{11}$ & Spanned by $s_{i}$ patterns \\
\hline$S_{1}$ & 1 & $\epsilon$ & 4 \\
$S_{2}$ & 1 & $-\epsilon$ & 5 \\
\hline$T^{\prime}: \overrightarrow{\mathrm{k}}=(\xi, 1-2 \xi, 0)$ \\
$M: \overrightarrow{\mathrm{k}}=(0.5,0,0)$ \\
\hline$M, T^{\prime}$ & $h_{1}$ & $h_{7}$ & Spanned by $s_{i}$ patterns \\
\hline$M_{1}, T_{1}^{\prime}$ & 1 & 1 & 4 \\
$M_{2}, T_{2}^{\prime}$ & 1 & -1 & 5 \\
\hline
\end{tabular}

${ }^{a} \epsilon=\exp \left(\frac{2}{3} i \pi\right)$.

${ }^{\mathrm{b}} \epsilon^{*}=\exp \left(-\frac{2}{3} i \pi\right)$.

$$
s_{i}=\frac{1}{g} \sum_{j=1}^{g} \chi^{(i) *}\left(R_{j}\right) \chi\left(R_{j}\right),
$$

where $g$ is the number of symmetry operations in the group of $\vec{k}, \chi\left(R_{j}\right)$ is the trace of the reduciblerepresentation matrix for the $j$ th operation, and $\chi^{(i) *}\left(R_{j}\right)$ is the complex conjugate of the trace (or character) found in the $i$ th irreducible representation of the $j$ th operation. $s_{i}$ is then the number of sets of symmetry coordinates, which span the irreducible representation.

The compatibility relations are obtained from a similar well-known relation ${ }^{9,10}$

$$
n_{i}=\frac{1}{g} \sum_{j=1}^{g} \chi^{(\mu) *}\left(R_{j}\right) \chi^{(\lambda)}\left(R_{j}\right),
$$

giving the number of times $n_{i}$ the $\mu$ th irreducible representation is contained in the $\lambda$ th irreducible representation corresponding to another symmetry direction in $\overrightarrow{\mathrm{k}}$ space. $\chi^{(\mu) *}\left(R_{j}\right)$ is the complex conjugate of the character for the $\mu$ th irreducible representation of the $j$ th operation, and $\chi^{(\lambda)}\left(R_{j}\right)$ is the character of the $\lambda$ th irreducible representation of the $j$ th operation.

From Eq. (3.14) and the character tables, we have found the compatibility relations listed in Table VIII.

Now it would be natural to ask if it was possible in some ingenious way to express the symmetry coordinates as analytical functions of the wave vector $\vec{k}$ in such a form that the same set may be 
TABLE VIII. Compatibility relations for various directions in reciprocal space of trigonal selenium.

\begin{tabular}{cccccccccccccc}
\hline \multicolumn{2}{c}{$(\Gamma, K) \rightarrow(\Delta, P) \rightarrow(A, H)$} & \multicolumn{3}{c}{$\Gamma \rightarrow T_{1} \rightarrow K$} & \multicolumn{3}{c}{$A \rightarrow S \rightarrow H$} & \multicolumn{3}{c}{$K \rightarrow T^{\prime} \rightarrow M$} \\
\hline$\Gamma_{1}, K_{1}$ & $\Delta_{1}, P_{1}$ & $A_{3}, H_{3}$ & $\Gamma_{1}$ & $T_{1}$ & $K_{1}$ & $A_{1}$ & $S_{1}$ & $H_{1}$ & $K_{1}$ & $T_{1}^{\prime}$ & $M_{1}$ \\
$\Gamma_{2}, K_{2}$ & $\Delta_{1}, P_{1}$ & $A_{3}, H_{3}$ & $\Gamma_{2}$ & $T_{2}$ & $K_{2}$ & $A_{2}$ & $S_{2}$ & $H_{2}$ & $K_{2}$ & $T_{2}^{\prime}$ & $M_{2}$ \\
$\Gamma_{2}, K_{2}$ & $\Delta_{1}, P_{1}$ & $A_{3}, H_{3}$ & $\Gamma_{2}$ & $T_{2}$ & $K_{2}$ & $A_{2}$ & $S_{2}$ & $H_{2}$ & $K_{2}$ & $T_{2}^{\prime}$ & $M_{2}$ \\
$\Gamma_{3}, K_{3}$ & $\Delta_{2}, P_{2}$ & $A_{3}, H_{3}$ & $\Gamma_{3}$ & $T_{1}$ & $K_{3}$ & $A_{3}$ & $S_{1}$ & $H_{3}$ & $K_{3}$ & $T_{1}^{\prime}$ & $M_{1}$ \\
$\Gamma_{3}, K_{3}$ & $\Delta_{3}, P_{3}$ & $A_{1}, H_{1}$ & $\Gamma_{3}$ & $T_{2}$ & $K_{3}$ & $A_{3}$ & $S_{2}$ & $H_{3}$ & $K_{3}$ & $T_{2}^{\prime}$ & $M_{2}$ \\
$\Gamma_{3}, K_{3}$ & $\Delta_{2}, P_{2}$ & $A_{3}, H_{3}$ & $\Gamma_{3}$ & $T_{1}$ & $K_{3}$ & $A_{3}$ & $S_{1}$ & $H_{3}$ & $K_{3}$ & $T_{1}^{\prime}$ & $M_{1}$ \\
$\Gamma_{3}, K_{3}$ & $\Delta_{3}, P_{3}$ & $A_{2}, H_{2}$ & $\Gamma_{3}$ & $T_{2}$ & $K_{3}$ & $A_{3}$ & $S_{2}$ & $H_{3}$ & $K_{3}$ & $T_{2}^{\prime}$ & $M_{2}$ \\
$\Gamma_{3}, K_{3}$ & $\Delta_{2}, P_{2}$ & $A_{3}, H_{3}$ & $\Gamma_{3}$ & $T_{1}$ & $K_{3}$ & $A_{3}$ & $S_{1}$ & $H_{3}$ & $K_{3}$ & $T_{1}^{\prime}$ & $M_{1}$ \\
$\Gamma_{3}, K_{3}$ & $\Delta_{3}, P_{3}$ & $A_{2}, H_{2}$ & $\Gamma_{3}$ & $T_{2}$ & $K_{3}$ & $A_{3}$ & $S_{2}$ & $H_{3}$ & $K_{3}$ & $T_{2}^{\prime}$ & $M_{2}$ \\
\hline \hline
\end{tabular}

used for all $\vec{k}$ vectors considered above. This would certainly be of great practical importance, as one only has to apply the projection approach once instead of performing the projection operation for each $\vec{k}$.vector analyzed. Such a set would also be easy to use in normal mode calculations providing a blocking of the dynamical matrix and a characterization of the symmetry properties of the modes for each $\vec{k}$, and it would display how symmetry coordinates of different symmetry merge into each other as $\vec{k}$ varies:

It turned out to be impossible to construct an analytical form for the symmetry coordinates valid for all considered directions. The most we could do was to construct sets, which may be used in each of the four directions listed in Table VIII. The sets from each direction are not convertible to each other. An equivalent conclusion was obtained from a study of the diamond structure.

In order to understand this, and in order to see how the projection should be performed to include as many $\vec{k}$ vectors as possible, let us for a moment return to the development given in Sec. II, Eqs. (2.13a) and (2.13b). There it was concluded that an $n$-dimensional matrix representing a symmetry operation in one basis in general is different from the matrix representing the same operation in another basis, generated from the first through linear combinations. However, if $n=1$ it is obvious that there is only one form of the "matrix" representing the operation. Therefore, a symmetry coordinate projected from a given displacement pattern of the atoms in the unit cell, will be given unambigously. For multidimensional representations there are a great deal of possibilities, as one may always change the representation matrices by any similarity transformation. Also, if a onedimensional representation is spanned by more than one symmetry coordinate, one may choose any linear combination of these to span the representation.

Suppose the objective is to construct symmetry coordinates for a given direction of the wave vector $\vec{k}$, then the projection should be done at a $|\vec{k}|$, where most of the irreducible representations are one dimensional. Symmetry coordinates spanning the one-dimensional representations will then be given unambigously, if the representations are spanned by only one coordinate. Coordinates spanning either multidimensional representations or the same one-dimensional representation are not given uniquely. However, going to other $|\vec{k}|$ in the given direction, other symmetries may occur (e.g., at the Brillouin zone or at the center of the Brillouin zone). It may now be possible to use the projected symmetry coordinates also at wave vectors of a symmetry different from the one considered by the projection, if suitable linear combinations of the coordinates, which were not determined unambigously, are considered. These linear combinations may be found from the compatibility relations, which display the transformation of the various symmetries, and the form of the symmetry coordinates at the particular $|\vec{k}|$, as determined by the projection-operator approach. Again, only in the presence of one-dimensional representations at the particular wave vector, one may have to consider specific linear combinations of the projected symmetry coordinates; it is therefore only necessary to project those symmetry coordinates, which span the one-dimensional representations at the new $|\vec{k}|$ in order to find the proper linear combinations. In this way symmetry coordinates for the four different directions listed in Table VIII were constructed. It turned out to be impossible to find a single form, which could be used for all directions simultaneously. Thus, for the directions $\Gamma \rightarrow \Delta-A$ and $\Gamma-T_{1} \rightarrow K$ it was impossible to find a common form of the symmetry coordinates due to a change in the coordinates, which span the two-dimensional $\Gamma_{3}$ representations, in going from the one direction to the other.

Let us illustrate the procedure for the direction $\Gamma \rightarrow \Delta-A$ and just list the results for the other 
three directions.

According to the recipe, we start at $\Delta$, where we have three one-dimensional representations each spanned by three symmetry coordinates. The projection operator $\hat{\boldsymbol{P}}$ for the three representations $\Delta_{1}, \Delta_{2}$, and $\Delta_{3}$ are obtained from (2.42), the representation of the symmetry operations and the character table. We find

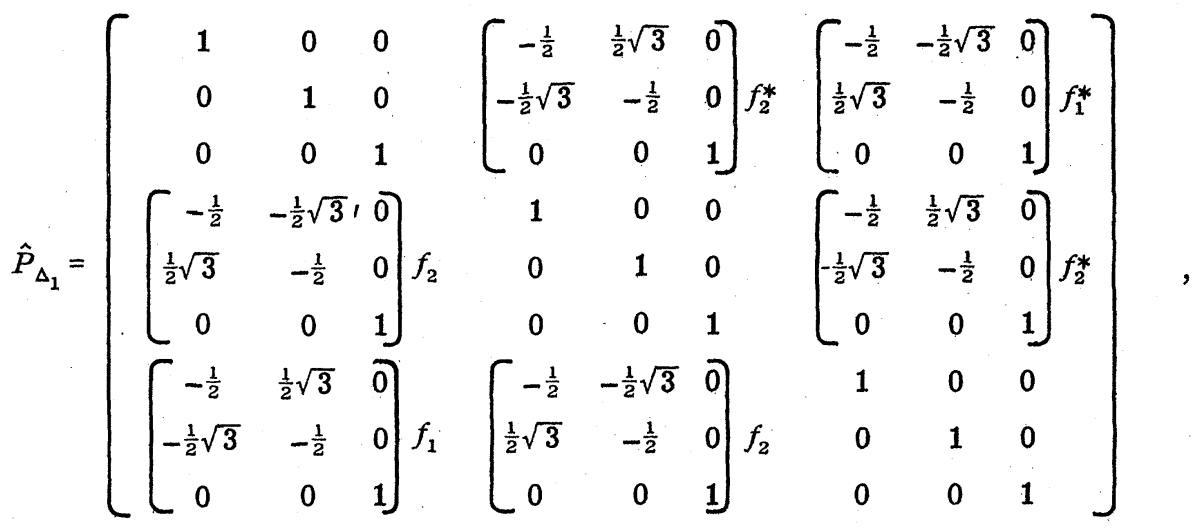

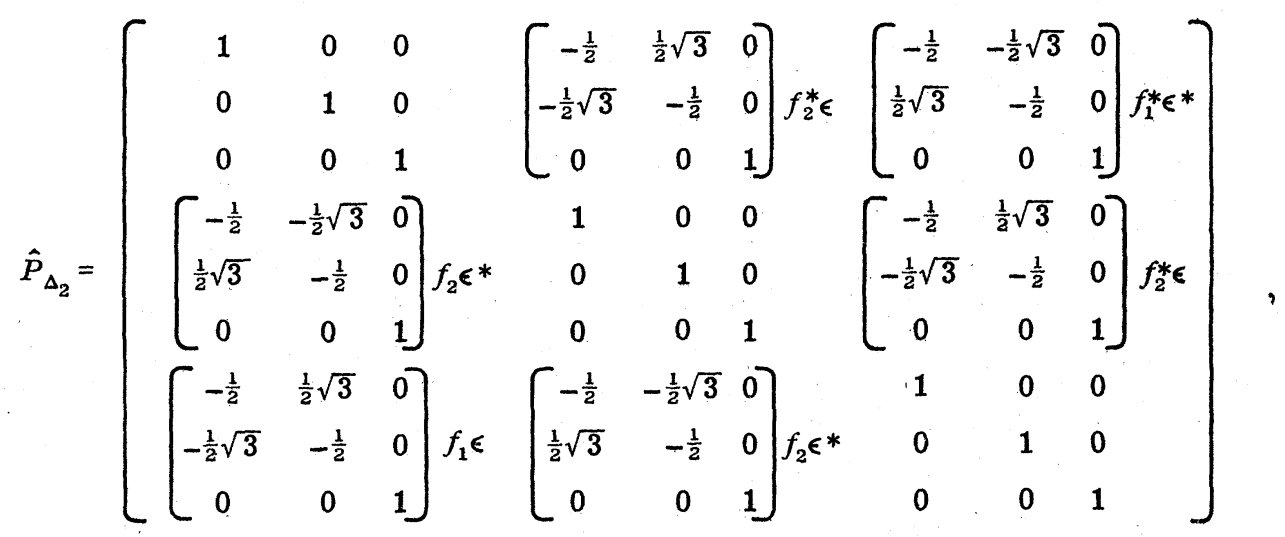

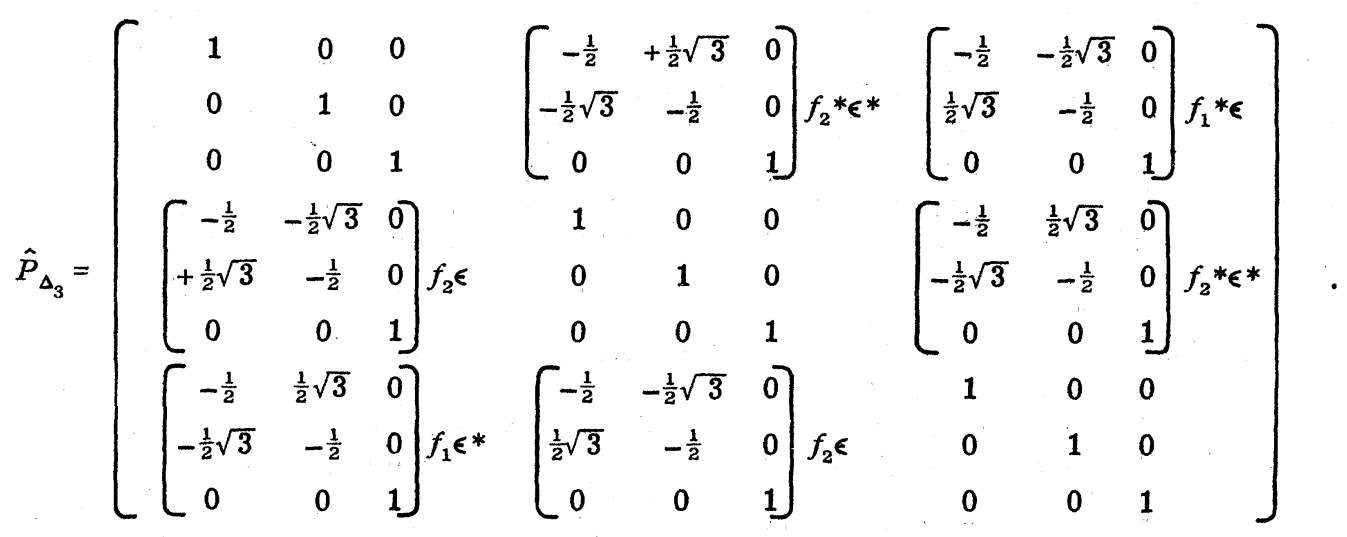


TABLE IX. Cartesian components of the linear-independent symmetry coordinates of selenium at $\Delta$ arranged in columns. Below each column the symmetry is indicated by a cross.

\begin{tabular}{cccccccccc}
\hline \hline & 1 & 0 & 0 & 1 & 0 & 0 & 1 & 0 & 0 \\
& 0 & 1 & 0 & 0 & 1 & 0 & 0 & 1 & 0 \\
& 0 & 0 & 1 & 0 & 0 & 1 & 0 & 0 & 1 \\
& $-\frac{1}{2} f_{2}$ & $-\frac{1}{2} \sqrt{3} f_{2}$ & 0 & $-\frac{1}{2} f_{2} \epsilon^{*}$ & $-\frac{1}{2} \sqrt{3} f_{2} \epsilon^{*}$ & 0 & $-\frac{1}{2} f_{2} \epsilon$ & $-\frac{1}{2} \sqrt{3} f_{2} \epsilon$ & 0 \\
& $\frac{1}{2} \sqrt{3} f_{2}$ & $-\frac{1}{2} f_{2}$ & 0 & $\frac{1}{2} \sqrt{3} f_{2} \epsilon^{*}$ & $-\frac{1}{2} f_{2} \epsilon^{*}$ & 0 & $\frac{1}{2} \sqrt{3} f_{2} \epsilon^{2}$ & $-\frac{1}{2} f_{2} \epsilon$ & 0 \\
& 0 & 0 & $f_{2}$ & 0 & 0 & $f_{2} \epsilon^{*}$ & 0 & 0 & $f_{2} \epsilon$ \\
& $-\frac{1}{2} f_{1}$ & $\frac{1}{2} \sqrt{3} f_{1}$ & 0 & $-\frac{1}{2} f_{1} \epsilon$ & $\frac{1}{2} \sqrt{3} f_{1} \epsilon$ & 0 & $-\frac{1}{2} f_{1} \epsilon^{*}$ & $\frac{1}{2} \sqrt{3} f_{1} \epsilon^{*}$ & 0 \\
& $-\frac{1}{2} \sqrt{3} f_{1}$ & $-\frac{1}{2} f_{1}$ & 0 & $-\frac{1}{2} \sqrt{3} f_{1} \epsilon$ & $-\frac{1}{2} f_{1} \epsilon$ & 0 & $-\frac{1}{2} \sqrt{3} f_{1} \epsilon^{*}$ & $-\frac{1}{2} f_{1} \epsilon^{*}$ & 0 \\
& 0 & 0 & $f_{1}$ & 0 & 0 & $f_{1} \epsilon$ & 0 & 0 & $f_{1} \epsilon^{*}$ \\
$\Delta_{1}$ & $\times$ & $\times$ & $\times$ & & & & & & \\
$\Delta_{3}$ & $\times$ & & & $\times$ & $\times$ & $\times$ & & & $\times$ \\
\hline \hline
\end{tabular}

To find a symmetry coordinate we let the projection operator $\hat{P}$ work on any display pattern $\underline{V}$ of the atoms in the unit cell, that is

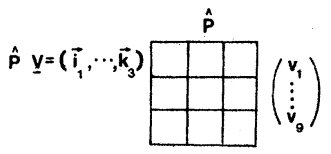

Since the set $\left(V_{1}, \ldots, V_{9}\right)$ is arbitrary, we let in turn $V_{i}=1, V_{j \neq i}=0(i, j=1, \ldots, 9)$. Then it is seen that the elements in each column of $\hat{P}$ may be considered as the components of the symmetry coordinates of the corresponding symmetry in the basis set of (3.9). The fact that there are more columns than symmetry coordinates simply means that some of the projected symmetry coordinates are linear combinations of others. From (3.13) we

TABLE X. Cartesian component of the normalized symmetry coordinates of Table IX arranged in columns. Below each column the symmetry is given at $\Gamma, \Delta$, and $A$.

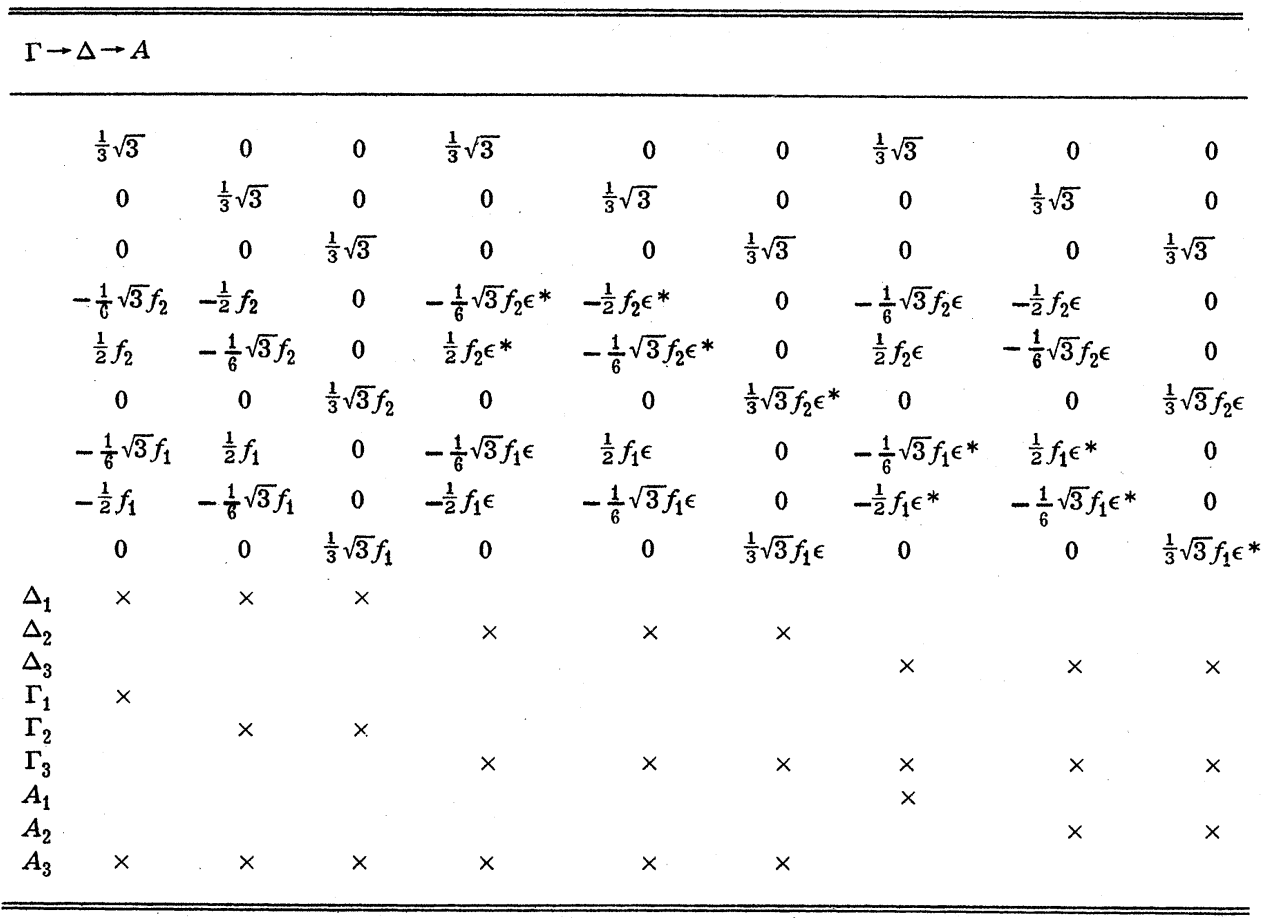


TABLE XI. Car components of the symmetry coordinates for the wave-vector direction $\Gamma \rightarrow T \rightarrow K$ arranged as in Table X.

\begin{tabular}{|c|c|c|c|c|c|c|c|c|c|}
\hline \multicolumn{10}{|c|}{$\Gamma \rightarrow T \rightarrow K$} \\
\hline & $\frac{1}{3} \sqrt{3}$ & 0 & 0 & $\frac{1}{6} \sqrt{6}$ & $\frac{1}{2} \sqrt{2}$ & 0 & 0 & 0 & 0 \\
\hline & 0 & $\frac{1}{2} \sqrt{2}$ & 0 & 0 & 0 & $\frac{1}{3} \sqrt{3}$ & 0 & $\frac{1}{6} \sqrt{6}$ & 0 \\
\hline & 0 & 0 & $\frac{1}{2} \sqrt{2}$ & 0 & 0 & 0 & $\frac{1}{3} \sqrt{3}$ & 0 & $\frac{1}{6} \sqrt{6}$ \\
\hline & $-\frac{1}{6} \sqrt{3}$ & $\frac{1}{4} \sqrt{6}$ & 0 & $-\frac{1}{12} \sqrt{6}$ & $\frac{1}{4} \sqrt{2}$ & $-\frac{1}{2}$ & 0 & $-\frac{1}{4} \sqrt{2}$ & 0 \\
\hline & $\frac{1}{2}$ & $\frac{1}{4} \sqrt{2}$ & 0 & $\frac{1}{4} \sqrt{2}$ & $-\frac{1}{4} \sqrt{6}$ & $-\frac{1}{6} \sqrt{3}$ & 0 & $-\frac{1}{12} \sqrt{6}$ & 0 \\
\hline & 0 & 0 & $-\frac{1}{2} \sqrt{2}$ & 0 & 0 & 0 & $\frac{1}{3} \sqrt{3}$ & 0 & $\frac{1}{6} \sqrt{6}$ \\
\hline & $-\frac{1}{6} \sqrt{3}$ & 0 & 0 & $\frac{1}{6} \sqrt{6}$ & 0 & $\frac{1}{2}$ & 0 & $-\frac{1}{2} \sqrt{2}$ & 0 \\
\hline & $-\frac{1}{2}$ & 0 & 0 & $\frac{1}{2} \sqrt{2}$ & 0 & $-\frac{1}{6} \sqrt{3}$ & 0 & $\frac{1}{6} \sqrt{6}$ & 0 \\
\hline & 0 & 0 & 0 & 0 & 0 & 0 & $\frac{1}{3} \sqrt{3}$ & 0 & $-\frac{1}{3} \sqrt{6}$ \\
\hline$T_{1}$ & $x$ & $x$ & $x$ & $\times$ & & & & & \\
\hline $\begin{array}{l}T_{2} \\
\Gamma_{1}\end{array}$ & $x$ & & & & $\times$ & $\times$ & $x$ & $\times$ & $x$ \\
\hline$\Gamma_{2}$ & & & & & & $x$ & $x$ & & \\
\hline $\begin{array}{l}\Gamma_{3} \\
K_{4}\end{array}$ & $x$ & $\times$ & $x$ & $x$ & $\times$ & & & $\times$ & $\times$ \\
\hline$K_{1}$ & & & & & & $x$ & $x$ & & \\
\hline$K_{3}$ & & $x$ & $x$ & $x$ & $\times$ & & & $x$ & $\times$ \\
\hline
\end{tabular}

TABLE XII. Cartesian components of the symmetry, coordinates for the wave-vector direction $A \rightarrow S \rightarrow H$ arranged as in Table X.

\begin{tabular}{|c|c|c|c|c|c|c|c|c|c|}
\hline \multicolumn{10}{|c|}{$A \rightarrow S \rightarrow H$} \\
\hline & $\frac{1}{3} \sqrt{3}$ & 0 & 0 & $\frac{1}{6} \sqrt{6}$ & $\frac{1}{2} \sqrt{2}$ & 0 & 0 & 0 & 0 \\
\hline & 0 & $\frac{1}{2} \sqrt{2}$ & 0 & 0 & 0 & $\frac{1}{3} \sqrt{3}$ & 0 & $\frac{1}{6} \sqrt{6}$ & 0 \\
\hline & 0 & 0 & $\frac{1}{2} \sqrt{2}$ & 0 & 0 & 0 & $\frac{1}{3} \sqrt{3}$ & 0 & $\frac{1}{6} \sqrt{6}$ \\
\hline & $\frac{1}{6} \sqrt{3}$ & $-\frac{1}{4} \sqrt{6}$ & 0 & $\frac{1}{12} \sqrt{6}$ & $-\frac{1}{4} \sqrt{2}$ & $\frac{1}{2}$ & 0 & $\frac{1}{4} \sqrt{2}$ & 0 \\
\hline & $-\frac{1}{2}$ & $-\frac{1}{4} \sqrt{2}$ & 0 & $-\frac{1}{4} \sqrt{2}$ & $\frac{1}{4} \sqrt{6}$ & $\frac{1}{6} \sqrt{3}$ & 0 & $\frac{1}{12} \sqrt{6}$ & 0 \\
\hline & 0 & 0 & $\frac{1}{2} \sqrt{2}$ & 0 & 0 & 0 & $-\frac{1}{3} \sqrt{3}$ & 0 & $-\frac{1}{6} \sqrt{6}$ \\
\hline & $-\frac{1}{6} \sqrt{3}$ & 0 & 0 & $\frac{1}{6} \sqrt{6}$ & 0 & $\frac{1}{2}$ & 0 & $-\frac{1}{2} \sqrt{2}$ & 0 \\
\hline & $-\frac{1}{2}$ & 0 & 0 & $\frac{1}{2} \sqrt{2}$ & 0 & $-\frac{1}{6} \sqrt{3}$ & 0 & $\frac{1}{6} \sqrt{6}$ & 0 \\
\hline & 0 & 0 & 0 & 0 & 0 & 0 & $\frac{1}{3} \sqrt{3}$ & 0 & $-\frac{1}{3} \sqrt{6}$ \\
\hline$S_{1}$ & $x$ & $x$ & $x$ & $x$ & & & & & \\
\hline $\begin{array}{l}S_{2} \\
A_{1}\end{array}$ & $x$ & & & & $x$ & $x$ & $x$ & $x$ & $x$ \\
\hline$A_{2}$ & & & & & & $x$ & $x$ & & \\
\hline $\begin{array}{l}A_{3} \\
H_{1}\end{array}$ & $x$ & $\times$ & $\times$ & $x$ & $\times$ & & & $x$ & $\times$ \\
\hline $\mathrm{H}_{2}$ & & & & & & $x$ & $\times$ & & \\
\hline $\mathrm{H}_{3}$ & & $x$ & $x$ & $\times$ & $\times$ & & & $x$ & $\times$ \\
\hline
\end{tabular}


TABLE XIII. Cartesian components of the symmetry coordinates for the wave-vector direction $K \rightarrow T^{\prime} \rightarrow M$ arranged as in Table $\mathrm{X}$.

\begin{tabular}{|c|c|c|c|c|c|c|c|c|c|}
\hline \multicolumn{10}{|c|}{$K \rightarrow T^{\prime} \rightarrow M$} \\
\hline & $\frac{1}{2} \sqrt{3}$ & 0 & 0 & $\frac{1}{6} \sqrt{6}$ & $\frac{1}{2} \sqrt{2}$ & 0 & 0 & 0 & 0 \\
\hline & 0 & $\frac{1}{2} \sqrt{2}$ & 0 & 0 & 0 & $\frac{1}{3} \sqrt{3}$ & 0 & $\frac{1}{6} \sqrt{6}$ & 0 \\
\hline & 0 & 0 & $\frac{1}{2} \sqrt{2}$ & 0 & 0 & 0 & $\frac{1}{3} \sqrt{3}$ & 0 & $\frac{1}{6} \sqrt{6}$ \\
\hline & $-\frac{1}{6} \sqrt{3}$ & 0 & 0 & $\frac{1}{6} \sqrt{6}$ & 0 & $-\frac{1}{2}$ & 0 & $\frac{1}{2} \sqrt{2}$ & 0 \\
\hline & $\frac{1}{2}$ & 0 & 0 & $-\frac{1}{2} \sqrt{2}$ & 0 & $-\frac{1}{6} \sqrt{3}$ & 0 & $\frac{1}{6} \sqrt{6}$ & 0 \\
\hline & 0 & 0 & 0 & 0 & 0 & 0 & $\frac{1}{3} \sqrt{3}$ & 0 & $-\frac{1}{3} \sqrt{6}$ \\
\hline & $-\frac{1}{6} \sqrt{3}$ & $-\frac{1}{4} \sqrt{6}$ & 0 & $-\frac{1}{12} \sqrt{6}$ & $\frac{1}{4} \sqrt{2}$ & $\frac{1}{2}$ & 0 & $\frac{1}{4} \sqrt{2}$ & 0 \\
\hline & $-\frac{1}{2}$ & $\frac{1}{4} \sqrt{2}$ & 0 & $-\frac{1}{4} \sqrt{2}$ & $\frac{1}{4} \sqrt{6}$ & $-\frac{1}{6} \sqrt{3}$ & 0 & $-\frac{1}{12} \sqrt{6}$ & 0 \\
\hline & 0 & 0 & $-\frac{1}{2} \sqrt{2}$ & 0 & 0 & 0 & $\frac{1}{3} \sqrt{3}$ & 0 & $\frac{1}{6} \sqrt{6}$ \\
\hline$T_{1}^{\prime}$ & $x$ & $x$ & $\times$ & $x$ & & & & & \\
\hline $\begin{array}{c}T_{2}^{\prime} \\
K_{1}\end{array}$ & $x$ & & & & $\times$ & $\times$ & $x$ & $x$ & $x$ \\
\hline $\begin{array}{l}K_{1} \\
K_{2}\end{array}$ & $\lambda$ & & & & & $x$ & $x$ & & \\
\hline$K_{3}$ & & $x$ & $x$ & $x$ & $x$ & & & $x$ & $x$ \\
\hline$M_{1}$ & $x$ & $x$ & $\times$ & $\times$ & & & & & \\
\hline$M_{2}$ & & & & & $\times$ & $x$ & $\times$ & $x$ & $x$ \\
\hline
\end{tabular}

know that there should be three linear independent symmetry coordinates of each symmetry, and they are easily found and listed in Table IX. This set of symmetry coordinates works at $\Delta$ and the question is whether it also will work at $\Gamma$ and $A$. From the compatibility relations is seen that $\Delta_{1} \rightarrow \Gamma_{1}$, and since $\Gamma_{1}$ is a one-dimensional representation spanned by only one symmetry coordinate, we have to choose a specific linear combination of the projected $\Delta_{1}$ symmetry coordinates, as the $\Gamma_{1}$ coordinate is given unambigously. The same situation exists with respect to the $\Delta_{3} \rightarrow A_{1}$ compatibility relation. As all $\Delta_{2}$ symmetries merge into the twodimensional $A_{3}$ and $\Gamma_{3}$ representations, there is no guide for choosing a specific linear combination of the $\Delta_{2}$ symmetry coordinates, so they can be used as they are. To find the specific linear combinations of the $\Delta_{1}$ symmetry coordinates, we just project the $\Gamma_{1}$ coordinate in the same way as before, and from that deduce which combination to consider.
It turns out that the $\Delta_{1}$ coordinate in the first column just has the $\Gamma_{1}$ symmetry at $\Gamma$ while the $\Delta_{3}$ coordinate in the seventh column just has the $A_{1}$ symmetry at $A$, so we need not consider any linear combination of the coordinates; they will work as they are. In other cases one has to consider linear combinations in order to achieve the desired result. This is for example the case for the other directions, but we will just list the results in Tables X-XIII. These results may be checked against the results of Teuchert et al., ${ }^{15}$ who also have generated symmetry coordinates of trigonal selenium.

\section{ACKNOWLEDGMENT}

The author wishes to express his gratitude to Dr. H. L. McMurry, Department of Physics, Idaho State University, Pocatello, Idaho for stimulating discussions in connection with the present work.
${ }^{1}$ F. Yssing Hansen, Comp. Phys. Commun. (to be published).

${ }^{2}$ H. L. McMurry and F. Yssing Hansen (unpublished). ${ }^{3}$ G. Herzberg, Molecular Spectra and Molecular Structure (Van Nostrand, New York, 1968).

${ }^{4}$ A. A. Maradudin and S. H. Vosko, Rev. Mod. Phys. $\underline{40}$,
1-37 (1968).

${ }^{5}$ J. L. Warren, Rev. Mod. Phys. 40, 38-76 (1968).

${ }^{6}$ A. C. Hurley, Philos. Trans. R. Soc. A 260, 1 (1966).

${ }^{7} \mathrm{O}$. V. Kovalev, Irreducible Representations of the Space Groups (Gordon and Breach, New York, 1965).

${ }^{8}$ G. K. Koster, in Solid State Physics, edited by 
F. Seitz and D. Turnbull (Academic, New York, 1957), Vol. 5.

${ }^{9} \mathrm{M}$. Tinkham, Group Theory and Quantum Mechanics (McGraw-Hill, New York, 1964).

${ }^{10}$ T. Janssen, Crystallographic Groups (North-Holland, Amsterdam, 1973).

${ }^{11} \mathrm{P}$. W. Atkins, Molecular Quantum Mechanics (Clarendon, Oxford, 1970).

${ }^{12}$ T. G. Worlton and J. L. Warren, Comp. Phys. Commun.
3, 88-117 (1972).

${ }^{13} \mathrm{~J}$. P. Dahl, Lectures on Group Theory, 1970 in Danish (unpublished), may be obtained from J. P. Dahl, Kemisk Laboratorium B, DTH 301, DK 2800, Lyngby, Denmark.

${ }^{14}$ L. I. Schiff, Quantum Mechanics (McGraw-Hill, New York, 1968).

${ }^{15}$ W. D. Teuchert and R. Geick, Phys. Solids 61,123 (1976). 